\title{
Are luminous radio-loud active galactic nuclei triggered by galaxy interactions?
}

\author{
C. Ramos Almeida, ${ }^{1 \star}$ P. S. Bessiere,${ }^{1}$ C. N. Tadhunter,${ }^{1}$ P. G. Pérez-González, ${ }^{2,3}$ \\ G. Barro, ${ }^{2}$ K. J. Inskip, ${ }^{4}$ R. Morganti, ${ }^{5,6}$ J. Holt $^{7}$ and D. Dicken ${ }^{8}$ \\ ${ }^{1}$ Department of Physics and Astronomy, University of Sheffield, Sheffield S3 7RH \\ ${ }^{2}$ Departamento de Astrofísica, Facultad de CC. Físicas, Universidad Complutense de Madrid, E-28040 Madrid, Spain \\ ${ }^{3}$ Associate Astronomer at Steward Observatory, The University of Arizona, 933 N Cherry Avenue, Tucson, AZ 85721, USA \\ ${ }^{4}$ Max-Planck-Institut für Astronomie, Köningstuhl 17, D-69117 Heidelberg, Germany \\ ${ }^{5}$ Netherlands Institute for Radio Astronomy, Postbus 2, 7990 AA Dwingeloo, the Netherlands \\ ${ }^{6}$ Kapteyn Astronomical Institute, University of Groningen, Postbus 800, 9700 AV Groningen, the Netherlands \\ ${ }^{7}$ Leiden Observatory, Leiden University, PO Box 9513, 2300 RA Leiden, the Netherlands \\ ${ }^{8}$ Institut d'Astrophysique Spatiale, CNRS, Université Paris Sud, 91405 Orsay, France
}

Accepted 2011 August 29. Received 2011 August 16; in original form 2011 July 14

\begin{abstract}
We present the results of a comparison between the optical morphologies of a complete sample of 46 southern 2 Jy radio galaxies at intermediate redshifts $(0.05<z<0.7)$ and those of two control samples of quiescent early-type galaxies: 55 ellipticals at redshifts $z \leq 0.01$ from the Observations of Bright Ellipticals at Yale (OBEY) survey, and 107 early-type galaxies at redshifts $0.2<z<0.7$ in the Extended Groth Strip (EGS). Based on these comparisons, we discuss the role of galaxy interactions in the triggering of powerful radio galaxies (PRGs). We find that a significant fraction of quiescent ellipticals at low and intermediate redshifts show evidence for disturbed morphologies at relatively high surface brightness levels, which are likely the result of past or on-going galaxy interactions. However, the morphological features detected in the galaxy hosts of the PRGs (e.g. tidal tails, shells, bridges, etc.) are up to 2 mag brighter than those present in their quiescent counterparts. Indeed, if we consider the same surface brightness limits, the fraction of disturbed morphologies is considerably smaller in the quiescent population (53 percent at $z<0.2$ and 48 percent at $0.2 \leq z<0.7$ ) than in the PRGs (93 per cent at $z<0.2$ and 95 per cent at $0.2 \leq z<0.7$ considering strong-line radio galaxies only). This supports a scenario in which PRGs represent a fleeting active phase of a subset of the elliptical galaxies that have recently undergone mergers/interactions. However, we demonstrate that only a small proportion $(\lesssim 20$ per cent) of disturbed early-type galaxies are capable of hosting powerful radio sources.
\end{abstract}

Key words: galaxies: active - galaxies: elliptical and lenticular, $\mathrm{cD}$ - galaxies: evolution galaxies: interactions - galaxies: nuclei.

\section{INTRODUCTION}

Simulations of hierarchical galaxy evolution predict that the periods of black hole $(\mathrm{BH})$ growth and nuclear activity are intimately tied to the growth of the host galaxy, and that the triggering of the main phase of this nuclear activity in gas-rich mergers will always be accompanied by a major galaxy-wide starburst (Kauffmann \& Haehnelt 2000; Di Matteo, Springel \& Hernquist 2005; Springel, Di Matteo \& Hernquist 2005; Hopkins et al. 2008a,b; Somerville et al. 2008). However, the order of events and the time-scales involved in both the triggering of the merger-induced starburst and the nuclear

^E-mail: c.ramos@sheffield.ac.uk activity remain uncertain (see e.g. Canalizo \& Stockton 2000; Wild, Heckman \& Charlot 2010; Tadhunter et al. 2011).

Based on cosmological simulations, Hopkins et al. (2008b) suggested a bimodality in the $\mathrm{BH}$ triggering mechanisms: luminous quasar-like activity is associated with the formation of classical bulges and ellipticals via galaxy mergers, whereas the less luminous Seyfert-like activity is associated with the formation of pseudo-bulges and bulgeless galaxies via secular processes. Under the assumption that major, gas-rich mergers trigger quasar activity, Hopkins et al. (2008b) reproduce the observed quasar luminosity function from $z=0$ to $z=6$. They also compare with a secular model in which the nuclear activity is driven by bars or instabilities and show that, although these processes probably dominate at luminosities typical of Seyfert galaxies, they contribute very little to the $z \gtrsim 1$ quasar luminosity density. 
From an observational point of view, a bimodality in formation mechanisms (and hence in AGN triggering) is supported by the fact that whereas classical bulges and elliptical galaxies follow a close correlation between velocity dispersion and BH mass (Kormendy \& Richstone 1995; Magorrian et al. 1998; Gebhardt et al. 2000; Ferrarese \& Merritt 2000; Greene \& Ho 2006), bulgeless galaxies and those with pseudo-bulges show no clear evidence for such a correlation (Graham 2008; Hu 2008; Graham et al. 2011; Kormendy, Bender \& Cornell 2011).

Galaxy interactions are one of the most efficient mechanisms to transport the cold gas required to trigger and feed AGN to the centre of galaxies (Kauffmann \& Haehnelt 2000; Cox et al. 2006, 2008; Croton et al. 2006; Di Matteo et al. 2007) and many observational studies of powerful AGN (i.e. quasar-like) have revealed a high incidence of interaction signatures in their host galaxies (Heckman et al. 1986; Hutchings 1987; Smith \& Heckman 1989; Canalizo \& Stockton 2001; Canalizo et al. 2007; Bennert et al. 2008, Ramos Almeida et al. 2011, hereafter RA11). However, all these studies lack comparisons with appropriate samples of quiescent (i.e. nonactive) galaxies to confirm that the percentage of interacting systems in powerful AGN is larger than in the quiescent population. Indeed, based on high spatial resolution Hubble Space Telescope (HST) images of a sample of nearby radio galaxies and quasars, Dunlop et al. (2003) found evidence that their hosts are indistinguishable from quiescent ellipticals of similar mass. Moreover, for moderately luminous AGN (i.e. Seyfert galaxies, $L_{\text {bol }} \sim 10^{42}-10^{45} \mathrm{erg} \mathrm{s}^{-1}$ ) several studies find that the incidence of disturbed morphologies is not significantly enhanced over the general population (e.g. Malkan, Gorjian \& Tam 1998; Grogin et al. 2005; Gabor et al. 2009; Georgakakis et al. 2009; Cisternas et al. 2011, hereafter C11), although some others find the opposite result (e.g. Keel 1996; Kuo et al. 2008; Koss et al. 2010; Ellison et al. 2011).

In our previous work ( RA11) we studied the optical morphologies of a complete sample of 46 southern 2 Jy radio galaxies at intermediate redshifts $(0.05<z<0.7)$ and found that the overall majority of the sample (up to 85 per cent) show peculiarities in their optical morphologies at relatively high levels of surface brightness. Our study indicates that galaxy interactions are likely to play a key role in the triggering of AGN/jet activity, especially in the case of strong-line radio galaxies (SLRGs), ${ }^{1}$ of which 94 per cent appear disturbed. On the other hand, of the weak-line radio galaxies (WLRGs) $^{2}$ in the 2 Jy sample, only 27 per cent show clear evidence for tidal features. These results are consistent with the most accepted explanation for the differences between the properties of SLRGs and WLRGs, in which SLRGs are powered by cold gas accretion, while WLRGs are fuelled by accretion of hot gas from their X-ray coronae (Allen et al. 2006; Best et al. 2006; Hardcastle, Evans \& Croston 2007; Balmaverde, Baldi \& Capetti 2008; Buttiglione et al. 2010).

The high percentage of disturbed morphologies in the 2 Jy sample of radio galaxies contrasts with the results found for lower luminosity AGN in, for example, the recent extensive study by $\mathrm{C} 11$. However, the RA11 and C11 studies can be reconciled by considering the differences in the depth of the observations and sample

\footnotetext{
${ }^{1}$ SLRGs comprise narrow-line radio galaxies (NLRGs), broad-line radio galaxies (BLRGs) and quasars, i.e. they are radio galaxies with strong and high equivalent width emission lines.

${ }^{2}$ WLRGs have optical spectra dominated by the stellar continua of the host galaxies and small emission line equivalent widths $\left(\mathrm{EW}_{[\mathrm{O} \mathrm{III]}}<10 \AA\right.$; Tadhunter et al. 1998).
}

selection. First, the images employed in $\mathrm{C} 11$ were obtained with the Advanced Camera for Surveys (ACS) on the HST and are not as deep as our Gemini Multi-Object Spectrograph South (GMOS-S)/ Gemini observations: the limiting surface brightness level of those HST images is $23.3 \mathrm{mag} \mathrm{arcsec}^{-2}$ in the ACS F814W filter, whereas in RA11 we detected features as faint as $26.3 \mathrm{mag} \mathrm{arcsec}^{-2}$ in the same band (using colour transformations for elliptical galaxies from Fukugita, Shimasaku \& Ichikawa 1995). Thus, the imaging observations used in $\mathrm{C} 11$, with a surface brightness limit 3 mag brighter than ours, are not sensitive enough to reveal faint diffuse tidal features in their AGN and control samples, even if present. Secondly, the AGN in C11 were selected at X-ray wavelengths and the galaxy hosts are mostly discs with luminosities more typical of luminous Seyfert galaxies (median $L_{\mathrm{bol}} \sim 10^{44.8} \mathrm{erg} \mathrm{s}^{-1}$ ). In contrast, the AGN in RA11 were selected according to their radio emission and the majority have quasar-like luminosities that are typically an order of magnitude higher than those of the $\mathrm{C} 11$ sample (median value of $L_{\mathrm{bol}}$ $\sim 10^{45.7} \mathrm{erg} \mathrm{s}^{-1}$ for the SLRGs in the $2 \mathrm{Jy} \mathrm{sample}^{3}$ ) and they are almost exclusively hosted by elliptical galaxies. Thus, the differences between the findings of RA11 and $\mathrm{C} 11$ can be explained by the luminosity-dependent bimodality in the $\mathrm{BH}$ triggering mechanisms suggested by Hopkins et al. (2008b) and Kormendy et al. (2011), as well as by the differences in depth between the observations employed.

If galaxy interactions are the main triggering mechanism for radio-loud AGN activity in our sample, then it is expected that the signs of morphological disturbance will be stronger and more common in the radio source host galaxies than in the general population of quiescent ellipticals. Studies of nearby red galaxies (e.g. van Dokkum 2005) have shown that the majority of quiescent luminous ellipticals were assembled through gas-poor mergers, which explains their old stellar populations and high central densities. However, triggering and feeding a powerful radio source (and occasionally star formation; see Tadhunter et al. 2011) is likely to require a larger amount of cold gas to be accreted into the central regions of the galaxy. The morphological signatures of gas-rich interactions (such as tidal tails, shells, bridges, etc.) are brighter than those produced in gas-poor interactions (Naab, Khochfar \& Burkert 2006; Bell et al. 2006; McIntosh et al. 2008). Thus, the surface brightness of the various morphological features can be used as an indicator of the type of interaction. In addition, the features resulting from gasrich interactions are expected to be visible over time-scales between 0.5 and 1.5 Gyr (Le Fèvre et al. 2000; Patton et al. 2002; Conselice et al. 2003; Kawata et al. 2006), whereas those formed in gas-poor interactions are visible for only $\sim 150 \mathrm{Myr}$ (Bell et al. 2006).

This is the second in a series of papers based on the analysis of the optical morphologies of powerful radio galaxies (PRGs). In the first paper (RA11), we presented deep Gemini images for the 2 Jy sample and compared the results found for the PRGs with various samples of quiescent ellipticals and/or red galaxies from the literature (Malin \& Carter 1983; van Dokkum 2005; Tal et al. 2009). However, only the observations reported in the study of Malin \& Carter (1983), which is based on photographic plates, have similar surface brightness depth to our PRG sample $\left(\mu_{V} \lesssim 25.5 \mathrm{mag} \operatorname{arcsec}^{-2}\right)$. After comparing with the latter study we concluded that the percentage of morphological disturbance of the PRGs (up to 85 per cent) greatly exceeds that found for quiescent elliptical galaxies when the same

\footnotetext{
${ }^{3} L_{\text {bol }}$ was derived from the [O III] luminosities of the individual galaxies listed in Dicken et al. (2009) by applying the bolometric correction factor of 3500 reported in Heckman et al. (2004) for low-redshift quasars.
} 
surface brightness depth is considered ( $\sim 10$ per cent). However, in order to make a more quantitative comparison, it is necessary to develop control samples of elliptical galaxies at similar redshifts and masses, probing the same scales and depths, and using CCD imaging data. In this paper we present the results from such a comparison. In Section 2 we describe the control sample selection and observations. In Section 3 we present the observational results. The comparison between the morphologies of PRGs and quiescent elliptical galaxies is discussed in Section 4, and the main conclusions from this work are summarized in Section 5. Throughout this paper we assume a cosmology with $H_{0}=70 \mathrm{~km} \mathrm{~s}^{-1} \mathrm{Mpc}^{-1}, \Omega_{\mathrm{m}}=0.27$ and $\Omega_{\Lambda}=0.73$.

\section{SAMPLE SELECTION AND OBSERVATIONS}

The objects studied in RA11 comprise all PRGs and quasars from the Tadhunter et al. (1993) sample of 2 Jy radio galaxies with $S_{2.7 \mathrm{GHz}} \geq 2.0 \mathrm{Jy}$, steep radio spectra $\alpha_{2.7}^{4.8}>0.5\left(F_{v} \propto v^{-\alpha}\right)$, declinations $\delta<+10^{\circ}$ and redshifts $0.05<z<0.7$ (see table 1 in RA11). It is itself a subset of the Wall \& Peacock (1985) complete sample of 2 Jy radio sources (see di Serego Alighieri et al. 1994 for further discussion on the sample completeness). The $z>0.05$ limit ensures that the radio galaxies are genuinely powerful sources, while the $z<0.7$ limit ensures that sources are sufficiently nearby for detailed morphological studies.

In terms of the optical classification, the sample comprises 24 percent WLRGs and 76 percent SLRGs (Tadhunter et al. 1998). Considering the radio morphologies, Fanaroff-Riley type II (FRII) sources constitute the majority of the sample (72 per cent), 13 percent are Fanaroff-Riley type I (FRI), and the remaining 15 per cent are compact, steep-spectrum (CSS) or Gigahertz-peaked spectrum (GPS) sources (see table 1 in RA11).

Moderately luminous AGN (e.g. those studied in C11) have a relatively high surface density and can be easily selected in deep field surveys, together with appropriate control samples of quiescent galaxies. In contrast, quasars and radio galaxies are much rarer and cannot be studied using narrow, deep field surveys. In consequence, it is more challenging to develop control samples for such objects.

Since radio galaxies are almost invariably associated with elliptical hosts (see e.g. Heckman et al. 1986; Dunlop et al. 2003), we searched in the literature for samples of elliptical galaxies with similar masses and redshifts as our 2 Jy PRGs. In addition, similar angular resolutions and depths are required to probe the same spatial scales and surface brightness levels. Our sample of 46 PRGs was imaged with the GMOS-S on the 8.1-m Gemini South telescope at Cerro Pachón under good seeing conditions (median seeing of 0.8 arcsec, ranging from 0.4 to 1.15 arcsec). The GMOS-S detector (Hook et al. 2004) comprises three adjacent CCDs, giving a field-of-view (FOV) of $5.5 \times 5.5 \mathrm{arcmin}^{2}$, with a pixel size of 0.146 arcsec. The morphological features reported in RA11 have a median surface brightness of $\mu_{V}=23.6 \mathrm{mag} \operatorname{arcsec}^{-2}$ and $\Delta \mu_{V} \sim[21$, 26] mag $\operatorname{arcsec}^{-2}$. See RA11 for a more detailed description of the GMOS-S observations. Thus, after considering all these factors, we finally selected control samples of elliptical galaxies in two redshift ranges which best match the 2 Jy sample host galaxies: the Observations of Bright Ellipticals at Yale (OBEY) survey and the Extended Groth Strip (EGS) sample.

\subsection{The OBEY survey}

The OBEY survey (Tal et al. 2009) is a volume-limited and statistically complete sample of 55 luminous elliptical galaxies selected from the Nearby Galaxies Catalogue (Tully 1988; see Table 1). It consists of all elliptical galaxies in the Tully (1988) catalogue with declinations between -85 and +10 , at distances from 16 to $40 \mathrm{Mpc}$, and $M_{B}<-20.4 \mathrm{mag}$, once corrected to the cosmology considered here. The sample comprises galaxies from a wide range of different environments: 36 per cent are field galaxies, 33 per cent are in groups and 18 per cent are in clusters, including members of the Virgo, Fornax, Centaurus and Antlia clusters. These galaxy environments were determined from the literature by Tal et al. (2009) and no classification is reported in that study for the remaining 13 per cent. This wide variety of environments in the OBEY survey matches those typical of FRII radio galaxies, which are found in field/groups as well as in moderately rich clusters (Prestage \& Peacock 1988; Smith \& Heckman 1990; Zirbel 1997) and constitute the majority of our PRG sample (72 per cent).

Thus, we have a sample of 55 giant elliptical galaxies at redshifts $z \leq 0.01$ and with absolute magnitudes $M_{B}=[-22.5,-20.4]$ mag. If we assume no evolution for massive elliptical galaxies since $z=$ 0.2 (Cimatti, Daddi \& Renzini 2006; Faber et al. 2007), we can compare the OBEY sample with the PRGs in the 2 Jy sample at $z<0.2$. In Fig. 1 we show a comparison between the absolute magnitudes of the 24 PRGs with $z<0.2$ and the 55 quiescent ellipticals from Tal et al. (2009). The $M_{B}$ values for the PRGs have been calculated from the Galactic extinction-, cosmological dimming- and $k$-corrected $r^{\prime}$-band magnitudes reported in RA11. Colours of elliptical galaxies from Fukugita et al. (1995) have been used to convert the magnitudes to the $B$ band, resulting in absolute magnitudes within the interval $M_{B}=[-22.1,-20.3]$ mag. From the comparison between the two $M_{B}$ distributions shown in Fig. 1, the significance level of the Kolmogorov-Smirnov (KS) statistic is 0.04 (i.e. there is only a 4 per cent chance that the two distributions are drawn from the same parent population). The low value of the KS probability is due to the larger number of $M_{B}>-21 \mathrm{mag}$ ellipticals in the OBEY sample compared to the PRGs. However, both distributions span the same range in absolute magnitude and we prefer to keep the complete sample of 55 quiescent elliptical galaxies rather than reducing the number of fainter objects. In addition, as we discuss in Section 3.1.3, we do not find any significant correlation between luminosity and the level of morphological disturbance.

The 55 galaxies in the OBEY survey were imaged with Y4KCam, which is a $4 \mathrm{~K} \times 4 \mathrm{~K}$ CCD camera optimized for wide-field broadband imaging mounted on the 1-m Small and Moderate Aperture Research Telescope System (SMARTS) telescope at the Cerro Tololo Inter-American Observatory (CTIO) between 2006 and 2009. The final images are a combination of several pointings of $300 \mathrm{~s}$ in the $V$ band, resulting in very deep frames with exposure times between 4200 and $7200 \mathrm{~s}$. Tal et al. (2009) reported a detection threshold of $\mu_{V} \sim 27.7 \mathrm{mag}$ (in this work we have measured a surface brightness of $\mu_{V}=28.2 \mathrm{mag} \mathrm{arcsec}^{-2}$ for the faintest feature detected, as shown in Section 3.1.4).

The data were binned in order to improve the signal-to-noise ratio of the images, which have a final pixel size of 1.156 arcsec and a typical value of the seeing of $\sim 1.7$ arcsec. More details on the sample selection and observations can be found in Tal et al. (2009) and are summarized in Table 1. The images are then deeper than the GMOS-S images of the PRGs in the 2 Jy sample, for which the faintest detected feature has a $\mu_{V}=26.2 \mathrm{mag} \mathrm{arcsec}^{-2}$, and both the pixel size and the average seeing are larger than those of the 2 Jy radio galaxies $\left(0.146\right.$ arcsec pixel $^{-1}$ and FWHM $\sim 0.8$ arcsec, respectively, for the GMOS-S images). However, considering that the galaxies in the OBEY sample are at a median distance of $36 \mathrm{Mpc}$ (spatial scale of $\sim 170 \mathrm{pc} \operatorname{arcsec}^{-1}$ ) and the PRGs (those 
Table 1. Full classification of the OBEY survey ordered by RA. Columns 2, 3 and 4 list RA, declination and spectroscopic redshift as reported in the NASA/IPAC Extragalactic Data base (NED). Columns 5, 6 and 7 correspond to the $B$-band absolute magnitudes from Tully (1988) and corrected to $H_{0}=70 \mathrm{~km} \mathrm{~s}^{-1} \mathrm{Mpc}^{-1}$, the Vega $(B-V)$ colours within effective radius from Michard (2005) and de Vaucouleurs et al. (1991), and the dates of observation. Column 8 lists the tidal parameter reported in Tal et al. (2009). Columns 9 and 10 list our morphological classification (T: tail; F: fan; B: bridge; S: shell; D: dust feature; $2 \mathrm{~N}$ : double nucleus; 3N: triple nucleus; A: amorphous halo; I: irregular feature. Brackets indicate uncertain identification of a feature), and division in groups: (1) galaxy pair or group in tidal interaction; (2) galaxies showing T, F, S, D, A, I; (3) multiple nuclei (inside a $10 \mathrm{kpc}$ ); (4) galaxies with dust as the only detected feature, (5) isolated galaxies with no sign of interaction. Features with uncertain identification have not been considered in the statistics discussed in this study.

\begin{tabular}{|c|c|c|c|c|c|c|c|c|c|}
\hline Galaxy & RA & Dec. & $z$ & $M_{B}$ & $(B-V)$ & Obs. date & $T_{\mathrm{c}}$ & Morphology & Group \\
\hline NGC 0584 & 01:31:20.7 & $-06: 52: 05$ & 0.0060 & -20.98 & 0.92 & 2006-08-22 & 0.076 & $2 \mathrm{~S}, \mathrm{~B}$ & 1 \\
\hline NGC 0596 & $01: 32: 52.1$ & $-07: 01: 55$ & 0.0062 & -20.52 & 0.90 & $2006-08-23$ & 0.110 & $\mathrm{~S}, \mathrm{~F}$ & 2 \\
\hline NGC 0720 & 01:53:00.5 & $-13: 44: 19$ & 0.0058 & -20.68 & 0.96 & $2006-08-24$ & 0.079 & $2 \mathrm{~F}$ & 2 \\
\hline NGC 1199 & 03:03:38.4 & $-15: 36: 49$ & 0.0085 & -20.39 & 0.97 & $2006-01-25$ & 0.067 & $\ldots$ & 5 \\
\hline NGC 1209 & 03:06:03.0 & $-15: 36: 41$ & 0.0086 & -20.66 & 0.95 & $2006-01-26$ & 0.116 & $\mathrm{~T}, 3 \mathrm{~F},[\mathrm{D}]$ & 2 \\
\hline NGC 1399 & $03: 38: 29.1$ & $-35: 27: 03$ & 0.0047 & -20.64 & 0.98 & 2008-09-30 & 0.064 & $\ldots$ & 5 \\
\hline NGC 1395 & $03: 38: 29.8$ & $-23: 01: 40$ & 0.0057 & -20.59 & 0.94 & 2006-01-27 & 0.094 & $3 S$ & 2 \\
\hline NGC 1407 & 03:40:11.9 & $-18: 34: 49$ & 0.0059 & -21.32 & 0.93 & $2006-01-28$ & 0.083 & $\ldots$ & 5 \\
\hline NGC 2865 & $09: 23: 30.2$ & $-23: 09: 41$ & 0.0087 & -21.00 & 0.78 & 2006-03-28 & 0.193 & $3 \mathrm{~S}, 2 \mathrm{~T},[\mathrm{D}]$ & 2 \\
\hline NGC 2974 & $09: 42: 33.3$ & $-03: 41: 57$ & 0.0064 & -20.93 & 0.95 & $2006-02-05$ & 0.110 & $\mathrm{~S},[\mathrm{D}]$ & 2 \\
\hline NGC 2986 & $09: 44: 16.0$ & $-21: 16: 41$ & 0.0076 & -21.00 & 0.99 & 2006-01-30 & 0.045 & {$[\mathrm{~B}]$} & 5 \\
\hline NGC 3078 & $09: 58: 24.6$ & $-26: 55: 37$ & 0.0086 & -20.93 & 0.97 & 2006-01-31 & 0.103 & $\ldots$ & 5 \\
\hline NGC 3258 & $10: 28: 53.6$ & $-35: 36: 20$ & 0.0093 & -20.62 & 0.92 & 2006-02-01 & 0.123 & {$[2 \mathrm{~N}]$} & 5 \\
\hline NGC 3268 & 10:30:00.6 & $-35: 19: 32$ & 0.0093 & -20.58 & 0.96 & 2006-02-03 & 0.087 & $\mathrm{~S}$ & 2 \\
\hline NGC 3557B & 11:09:32.1 & $-37: 20: 59$ & 0.0096 & -20.43 & 0.86 & 2006-02-04 & 0.182 & $2 \mathrm{I}$ & 2 \\
\hline NGC 3557 & 11:09:57.6 & $-37: 32: 21$ & 0.0103 & -22.48 & 0.87 & 2006-01-31 & 0.111 & $\mathrm{~F},[\mathrm{~S}]$ & 2 \\
\hline NGC 3585 & $11: 13: 17.1$ & $-26: 45: 18$ & 0.0047 & -21.23 & 0.91 & 2006-03-29 & 0.048 & $2 \mathrm{~S}$ & 2 \\
\hline NGC 3640 & $11: 21: 06.8$ & $+03: 14: 05$ & 0.0041 & -21.08 & 0.92 & 2006-04-01 & 0.142 & $\mathrm{~S}, 4 \mathrm{~F}$ & 2 \\
\hline NGC 3706 & $11: 29: 44.4$ & $-36: 23: 29$ & 0.0099 & -21.44 & 0.93 & $2006-01-26$ & 0.120 & $2 \mathrm{~S}$ & 2 \\
\hline NGC 3904 & $11: 49: 13.2$ & $-29: 16: 36$ & 0.0052 & -20.41 & 0.94 & $2006-01-28$ & 0.108 & $\mathrm{~S}$ & 2 \\
\hline NGC 3923 & $11: 51: 01.8$ & $-28: 48: 22$ & 0.0058 & -21.53 & 0.95 & 2006-01-29 & 0.100 & $4 \mathrm{~S}$ & 2 \\
\hline NGC 3962 & $11: 54: 40.1$ & $-13: 58: 30$ & 0.0060 & -21.03 & 0.95 & 2006-03-30 & 0.059 & $\mathrm{~S}$ & 2 \\
\hline NGC 4105 & $12: 06: 40.8$ & $-29: 45: 37$ & 0.0064 & -20.66 & 0.87 & $2006-02-02$ & 0.109 & $2 \mathrm{~F}, \mathrm{~T}$ & 1 \\
\hline NGC 4261 & $12: 19: 23.2$ & $+05: 49: 31$ & 0.0074 & -21.71 & 0.98 & 2006-04-03 & 0.053 & $\mathrm{~T}, \mathrm{~F}$ & 2 \\
\hline NGC 4365 & $12: 24: 28.2$ & $+07: 19: 03$ & 0.0041 & -20.82 & 0.97 & 2006-03-29 & 0.070 & F & 2 \\
\hline IC 3370 & $12: 27: 37.3$ & $-39: 20: 16$ & 0.0097 & -21.53 & 0.89 & 2006-04-02 & 0.192 & F,S,D & 2 \\
\hline NGC 4472 & $12: 29: 46.7$ & $+08: 00: 02$ & 0.0033 & -22.12 & 0.97 & 2008-06-09 & 0.000 & $\ldots$ & 5 \\
\hline NGC 4636 & $12: 42: 49.9$ & $+02: 41: 16$ & 0.0031 & -20.98 & 0.93 & 2006-03-28 & 0.066 & F & 2 \\
\hline NGC 4645 & $12: 44: 10.0$ & $-41: 45: 00$ & 0.0087 & -21.18 & 0.95 & 2009-04-18 & 0.000 & $\ldots$ & 5 \\
\hline NGC 4697 & $12: 48: 35.9$ & $-05: 48: 03$ & 0.0041 & -21.97 & 0.92 & 2006-04-04 & 0.091 & $\ldots$ & 5 \\
\hline NGC 4696 & $12: 48: 49.3$ & $-41: 18: 40$ & 0.0098 & -22.35 & 0.94 & 2008-06-08 & 0.075 & S,D & 2 \\
\hline NGC 4767 & $12: 53: 52.9$ & $-39: 42: 52$ & 0.0099 & -21.43 & 0.93 & 2008-06-10 & 0.000 & $2 \mathrm{~S},[\mathrm{D}]$ & 2 \\
\hline NGC 5011 & $13: 12: 51.8$ & $-43: 05: 46$ & 0.0105 & -21.40 & 0.89 & 2006-04-05 & 0.077 & $\ldots$ & 5 \\
\hline NGC 5018 & $13: 13: 01.0$ & $-19: 31: 05$ & 0.0093 & -21.76 & 0.85 & 2008-06-03 & 0.184 & $3 \mathrm{~T}, 3 \mathrm{~S},[\mathrm{D}]$ & 2 \\
\hline NGC 5044 & $13: 15: 24.0$ & $-16: 23: 08$ & 0.0092 & -21.31 & 0.98 & 2008-06-06 & 0.041 & $\ldots$ & 5 \\
\hline NGC 5061 & $13: 18: 05.1$ & $-26: 50: 14$ & 0.0069 & -21.49 & 0.85 & 2006-04-01 & 0.104 & $\mathrm{~T}, \mathrm{~S}$ & 2 \\
\hline NGC 5077 & $13: 19: 31.7$ & $-12: 39: 25$ & 0.0093 & -20.82 & 0.98 & $2006-03-30$ & 0.061 & {$[\mathrm{~S}],[\mathrm{D}]$} & 5 \\
\hline NGC 5576 & $14: 21: 03.7$ & $+03: 16: 16$ & 0.0049 & -20.70 & 0.88 & 2008-06-06 & 0.122 & $3 \mathrm{~T}, \mathrm{~S}$ & 2 \\
\hline NGC 5638 & $14: 29: 40.4$ & $+03: 14: 00$ & 0.0055 & -21.42 & 0.94 & 2008-06-07 & 0.036 & $\mathrm{~T}, \mathrm{~S}$ & 2 \\
\hline NGC 5812 & $15: 00: 55.7$ & $-07: 27: 26$ & 0.0065 & -20.88 & 0.94 & 2008-06-08 & 0.080 & $\mathrm{~T}$ & 2 \\
\hline NGC 5813 & $15: 01: 11.2$ & $+01: 42: 07$ & 0.0065 & -21.07 & 0.95 & 2008-06-09 & 0.054 & $\ldots$ & 5 \\
\hline NGC 5846 & $15: 06: 29.3$ & $+01: 36: 20$ & 0.0057 & -21.46 & 0.98 & 2008-06-07 & 0.068 & $3 \mathrm{~S}, 2 \mathrm{~N}$ & 2,3 \\
\hline NGC 5898 & $15: 18: 13.5$ & $-24: 05: 53$ & 0.0070 & -20.79 & 0.92 & 2006-04-05 & 0.114 & $3 \mathrm{~T}, \mathrm{D}, 2 \mathrm{~N}$ & 2,3 \\
\hline NGC 5903 & $15: 18: 36.5$ & $-24: 04: 07$ & 0.0085 & -21.18 & 0.89 & 2006-04-05 & 0.075 & $\ldots$ & 5 \\
\hline IC 4797 & $18: 56: 29.7$ & $-54: 18: 21$ & 0.0089 & -21.05 & 0.92 & $2006-08-23$ & 0.226 & $\mathrm{~T}, \mathrm{I},[\mathrm{D}]$ & 2 \\
\hline IC 4889 & $19: 45: 15.1$ & $-54: 20: 39$ & 0.0085 & -20.85 & 0.88 & 2006-08-18 & 0.158 & $\mathrm{~F}$ & 2 \\
\hline NGC 6861 & $20: 07: 19.5$ & $-48: 22: 13$ & 0.0094 & -21.10 & 0.95 & 2006-08-19 & 0.123 & $\mathrm{D}$ & 4 \\
\hline NGC 6868 & 20:09:54.1 & $-48: 22: 46$ & 0.0095 & -21.36 & 0.97 & 2006-08-20 & 0.096 & $\ldots$ & 5 \\
\hline NGC 6958 & $20: 48: 42.6$ & $-37: 59: 51$ & 0.0090 & -20.83 & 0.86 & $2006-08-22$ & 0.122 & $3 \mathrm{~S},[\mathrm{D}]$ & 2 \\
\hline NGC 7029 & $21: 11: 52.0$ & $-49: 17: 01$ & 0.0094 & -20.41 & 0.86 & $2006-08-22$ & 0.085 & $\ldots$ & 5 \\
\hline NGC 7144 & $21: 52: 42.4$ & $-48: 15: 14$ & 0.0064 & -20.66 & 0.91 & 2006-08-17 & 0.100 & $\ldots$ & 5 \\
\hline NGC 7196 & $22: 05: 54.8$ & $-50: 07: 10$ & 0.0097 & -20.62 & 0.91 & 2006-08-19 & 0.171 & $\mathrm{~S},[\mathrm{D}]$ & 2 \\
\hline NGC 7192 & $22: 06: 50.1$ & $-64: 18: 58$ & 0.0099 & -20.85 & 0.92 & $2006-08-20$ & 0.096 & $\mathrm{~S}$ & 2 \\
\hline IC 1459 & $22: 57: 10.6$ & $-36: 27: 44$ & 0.0060 & -20.88 & 0.96 & 2006-08-18 & 0.137 & $4 \mathrm{~S}$ & 2 \\
\hline NGC 7507 & $23: 12: 07.6$ & $-28: 32: 23$ & 0.0052 & -20.51 & 0.94 & 2006-08-21 & 0.084 & $S$ & 2 \\
\hline
\end{tabular}



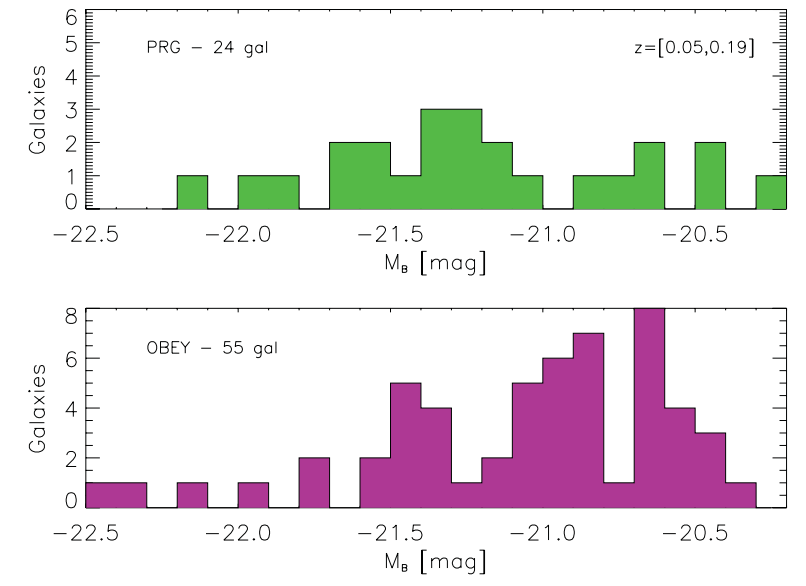

Figure 1. Comparison between the $B$-band absolute magnitudes of the PRGs in the 2 Jy sample at $z<0.2$ (top panel) and those of the Tal et al. (2009) sample of quiescent ellipticals at $z \leq 0.01$ (bottom panel).

at $z<0.2$ ) at $423 \mathrm{Mpc}$ (spatial scale of $\sim 1700 \mathrm{pc} \mathrm{arcsec}^{-1}$ ) the effective resolution will be better in the case of the OBEY survey images. Thus, considering that the latter images are deeper and the resolution better, we will likely detect fainter and smaller features than for the PRGs. Even in the case of large-scale diffuse structures, the fact that the OBEY images are 2 mag deeper than those of the 2 Jy sample will allow us to detect them. Summarizing, by using the same classification technique employed for the PRGs, we will be able to detect the same morphological signatures, if present, in the OBEY survey. Any possible bias will lead to a relative enhancement of the number of detected features in this sample relative to the PRG sample studied in RA11.

Since no observations of photometric standard stars were taken during the OBEY survey observations, we self-calibrated the images using aperture photometry measurements of the sample of elliptical galaxies from Prugniel \& Heraudeau (1998), as in Tal et al. (2009).

\subsection{The Extended Groth Strip sample}

In order to develop a control sample for the PRGs in the 2 Jy sample at redshifts $0.2 \leq z<0.7$ we have used the Rainbow Cosmological Surveys data base, ${ }^{4}$ which is a compilation of photometric and spectroscopic data, jointly with value-added products such as photometric redshifts, stellar masses, star formation rates and synthetic rest-frame magnitudes, for several deep cosmological fields (Pérez-González et al. 2008; Barro et al. 2009, 2011). Specifically, we have selected our control sample in the EGS $(\alpha=$ $\left.14^{\mathrm{h}} 17^{\mathrm{m}}, \delta=+52^{\circ} 30^{\prime}\right)$, which enlarges the HST Groth-Westphal strip (Groth et al. 1994) up to $2^{\circ} \times 15^{\prime}$ and has the advantage of being a low extinction area. We chose the EGS because it is a survey that covers sufficient area, and consequently enough galaxies, to extract statistically meaningful results, and because of the vast amount of public data available, including photometric redshifts, absolute magnitudes, colours and deep imaging in the optical (Davis et al. 2007). Indeed, here we use broad-band images in the $R_{\mathrm{c}}$ filter obtained with the Subaru Telescope, which are similar in pixel size and depth to the GMOS-S images of the PRGs employed in RA11.

\footnotetext{
${ }^{4}$ https://rainbowx.fis.ucm.es/Rainbow_Data base
}

Thus, we selected all the galaxies in the EGS with the same redshift and absolute magnitude ranges as the PRGs at $z \geq 0.2$ in RA11 $\left(0.2 \leq z<0.7\right.$ and $-22.2 \leq M_{B} \leq-20.6$ mag, respectively). The limiting values of this $M_{B}$ range were defined by considering NLRGs and WLRGs in RA11, since the quasars and BLRGs are likely to be contaminated by a large contribution from AGN emission. From this first selection we discard the sources in the EGS detected in X-rays (i.e. possible AGN) and foreground stars. The stars were automatically identified based on a combination of several criteria including their morphology (stellarity index) and their optical/NIR colours (see Pérez-González et al. 2008; Barro et al. 2011 for details on the star-galaxy separation criteria). In order to identify elliptical galaxies, we imposed a colour selection criterion: initially we selected all the sources with rest-frame colours $\left(M_{u}-\right.$ $\left.M_{g}\right)>1.5$, typical of galaxies located in the red sequence in the colour-magnitude diagram (Blanton 2006).

We choose this initial colour selection rather than morphologically selecting elliptical galaxies from the outset in order to avoid possible biases. The goal of this paper is to compare the morphologies of quiescent ellipticals with those of PRGs; by morphologically selecting elliptical galaxies (either by eye or automatically) we could be discarding highly disturbed sources, leading to a underestimation of interacting systems in the control sample. After applying the colour selection, we made a first visual classification of the sources into three groups: elliptical galaxies (E), possible discs (PD) and discs (D). According to Bundy et al. (2010), the red sequence is populated not only by elliptical and S0 galaxies, but also by early-type (ET) spirals. We then discarded all the galaxies that appeared as clear discs and kept the elliptical galaxies and PDs in the sample. The latter might include disturbed ellipticals that look more disc-like, or S0/ET spirals. After considering all these criteria, we have a control sample of 107 red ET galaxies in the EGS (see Table 2).

In Figs 2 and 3 we show the comparison in absolute magnitude and redshift between the PRGs and the EGS control sample. Note that in Fig. 2 we do not include the six BLRGs and quasars with $M_{B}<-22.2 \mathrm{mag}$. The EGS redshifts are photometric with an average quality of $\Delta z /(1+z)=0.03$. The absolute magnitudes were estimated by convolving the best-fitting galaxy templates, used to calculate the photometric redshifts, with the appropriate filter transmission (see Pérez-González et al. 2008; Barro et al. 2009, 2011 for specific details). The significance level of the KS statistic from the comparison between the two $M_{B}$ distributions shown in Fig. 2 is 0.18 , indicating that there is no significant difference between the two samples in terms of the distribution of absolute magnitude. The same is valid for the comparison between the redshift distributions (Fig. 3), for which the value of the KS probability is 0.15 .

The EGS was imaged with the Subaru Prime Focus Camera (Suprime-Cam; Miyazaki et al. 2002), which is a mosaic of ten $2048 \times 4096$ CCDs, located at the prime focus of the Subaru Telescope. Details on the observations of the EGS can be found in Zhao et al. (2009). It covers a $34 \times 27 \operatorname{arcmin}^{2}$ FOV with a pixel scale of 0.202 arcsec. The Suprime-Cam data consist of four $R_{c^{-}}$ band images of $1200 \mathrm{~s}$ exposure time that cover the entire field to a $5 \sigma$ limiting $\mathrm{AB}$ magnitude of 26.5 (Park et al. 2008). In this work we have measured a surface brightness of $\mu_{V}=26.3 \mathrm{mag}$ $\operatorname{arcsec}^{-2}$ for the faintest feature detected, as shown in Section 3.1.4. The seeing measured for the four images ranges from 0.60 to 0.72 arcsec. Thus, the data are comparable in depth and resolution to the GMOS-S images employed in the study of the morphologies 
Table 2. Full classification of the EGS sample ordered by Infrared Array Camera (IRAC) ID (Rainbow data base identifier). Columns 2, 3 and 4 list RA, declination and photometric redshift. Columns 5 and 6 correspond to the $B$-band absolute magnitudes and $(\mathrm{M} u-\mathrm{M} g)$ rest-frame colours from the Rainbow data base. Column 7 indicates whether a galaxy has been visually classified as an elliptical or as a PD. Columns 8 and 9 list the morphological classification and group as in Table 1. Features with uncertain identification (within brackets) have not been considered in the statistics discussed in this work.

\begin{tabular}{|c|c|c|c|c|c|c|c|c|}
\hline IRAC ID & RA & Dec. & $z_{\text {phot }}$ & $M_{B}$ & $(\mathrm{M} u-\mathrm{M} g)$ & Type & Morphology & Group \\
\hline 004162 & $14: 21: 29.6$ & $52: 58: 35$ & 0.48 & -20.65 & 1.75 & E & & 5 \\
\hline 006612 & $14: 17: 29.5$ & $52: 44: 33$ & 0.31 & -21.56 & 1.71 & E & $\mathrm{B}, \mathrm{F},[\mathrm{D}],[\mathrm{T}]$ & 1,3 \\
\hline 006613 & $14: 17: 29.3$ & $52: 44: 29$ & 0.30 & -20.81 & 1.70 & E & B & 1 \\
\hline 056690 & $14: 15: 14.4$ & $52: 12: 52$ & 0.50 & -21.01 & 1.70 & $\mathrm{E}$ & {$[\mathrm{A}],[\mathrm{B}]$} & 5 \\
\hline 060191 & $14: 23: 43.2$ & $53: 35: 33$ & 0.57 & -21.82 & 1.72 & $\mathrm{E}$ & $\mathrm{F}$ & 2 \\
\hline 060958 & $14: 23: 25.2$ & $53: 37: 59$ & 0.40 & -20.84 & 1.84 & PD & $\mathrm{T},[\mathrm{A}],[\mathrm{B}]$ & 1,2 \\
\hline 061249 & $14: 23: 34.8$ & $53: 35: 28$ & 0.65 & -21.95 & 1.68 & PD & [T] & 5 \\
\hline 066105 & $14: 23: 25.2$ & $53: 26: 14$ & 0.51 & -21.46 & 1.64 & $\mathrm{E}$ & [A] & 5 \\
\hline 067417 & $14: 23: 01.4$ & $53: 28: 33$ & 0.39 & -21.25 & 1.60 & E & $\ldots$ & 5 \\
\hline 072533 & $14: 22: 26.9$ & $53: 25: 19$ & 0.33 & -20.67 & 1.64 & E & $\mathrm{S}$ & 2 \\
\hline 073519 & $14: 22: 21.1$ & $53: 24: 32$ & 0.49 & -21.20 & 1.55 & E & {$[\mathrm{A}]$} & 5 \\
\hline 074777 & $14: 22: 24.5$ & $53: 21: 09$ & 0.42 & -21.91 & 1.61 & E & {$[\mathrm{S}]$} & 5 \\
\hline 074924 & $14: 22: 24.5$ & $53: 20: 53$ & 0.41 & -21.35 & 1.67 & $\mathrm{E}$ & $\ldots$ & 5 \\
\hline 077695 & $14: 22: 18.2$ & $53: 16: 39$ & 0.35 & -20.92 & 1.69 & PD & $\mathrm{T}$ & 2 \\
\hline 079968 & $14: 22: 02.4$ & $53: 15: 17$ & 0.60 & -21.62 & 1.82 & $\mathrm{E}$ & $\mathrm{F}$ & 2 \\
\hline 082325 & $14: 21: 22.8$ & $53: 18: 39$ & 0.55 & -22.05 & 1.82 & E & {$[\mathrm{F}]$} & 5 \\
\hline 083714 & $14: 21: 24.5$ & $53: 15: 34$ & 0.50 & -22.01 & 1.52 & E & $\mathrm{F}$ & 2 \\
\hline 088031 & $14: 21: 17.5$ & 53:09:09 & 0.50 & -21.04 & 1.68 & E & $\mathrm{F}$ & 2 \\
\hline 090430 & $14: 21: 09.1$ & $53: 07: 43$ & 0.38 & -21.83 & 1.75 & E & $\mathrm{A}, \mathrm{F},[\mathrm{B}]$ & 2 \\
\hline 092065 & $14: 21: 10.3$ & $53: 05: 40$ & 0.55 & -21.44 & 1.80 & $\mathrm{E}$ & B & 1 \\
\hline 092765 & $14: 20: 41.0$ & $53: 11: 01$ & 0.35 & -21.60 & 1.77 & PD & {$[\mathrm{A}],[\mathrm{T}]$} & 5 \\
\hline 093764 & $14: 20: 34.3$ & $53: 11: 23$ & 0.39 & -21.50 & 1.52 & $\mathrm{E}$ & {$[\mathrm{S}]$} & 5 \\
\hline 094231 & $14: 21: 00.0$ & $53: 05: 36$ & 0.41 & -21.98 & 1.81 & E & $2 \mathrm{~F},[\mathrm{~T}]$ & 2 \\
\hline 094966 & $14: 21: 21.1$ & $53: 00: 27$ & 0.46 & -21.65 & 1.76 & E & $2 \mathrm{~T}$ & 2 \\
\hline 095727 & $14: 20: 48.7$ & $53: 06: 24$ & 0.38 & -21.59 & 1.76 & $\mathrm{E}$ & $\mathrm{F}, \mathrm{S}$ & 2 \\
\hline 099954 & $14: 20: 14.4$ & $53: 09: 06$ & 0.27 & -20.83 & 1.71 & $\mathrm{E}$ & {$[\mathrm{T}]$} & 5 \\
\hline 102757 & $14: 19: 57.6$ & 53:09:40 & 0.22 & -20.81 & 1.71 & E & $2 S$ & 2 \\
\hline 102982 & $14: 20: 56.6$ & $52: 57: 14$ & 0.60 & -21.80 & 1.67 & E & F & 2 \\
\hline 103198 & $14: 20: 43.2$ & $52: 59: 46$ & 0.38 & -21.45 & 1.76 & $\mathrm{E}$ & $2 \mathrm{~N}, \mathrm{~F}, \mathrm{~S}$ & 2,3 \\
\hline 104038 & $14: 20: 29.3$ & $53: 01: 46$ & 0.46 & -20.72 & 1.75 & $\mathrm{E}$ & B & 1 \\
\hline 104729 & $14: 20: 43.0$ & $52: 58: 14$ & 0.63 & -21.76 & 1.61 & PD & A & 2 \\
\hline 105193 & $14: 20: 07.0$ & $53: 05: 07$ & 0.23 & -21.08 & 1.90 & $\mathrm{E}$ & {$[\mathrm{S}]$} & 5 \\
\hline 106324 & $14: 19: 48.0$ & $53: 07: 49$ & 0.26 & -20.85 & 1.60 & $\mathrm{E}$ & {$[\mathrm{T}]$} & 5 \\
\hline 106984 & $14: 20: 47.0$ & $52: 54: 57$ & 0.45 & -20.98 & 1.72 & PD & $\mathrm{A},[\mathrm{I}]$ & 2 \\
\hline 111427 & $14: 20: 23.5$ & $52: 55: 02$ & 0.32 & -20.81 & 1.66 & $\mathrm{E}$ & $2 \mathrm{~N}, \mathrm{~T}, 2 \mathrm{I}$ & 1,3 \\
\hline 112580 & $14: 20: 01.2$ & $52: 58: 23$ & 0.51 & -21.62 & 1.70 & $\mathrm{E}$ & [B] & 5 \\
\hline 113088 & $14: 20: 00.7$ & $52: 57: 57$ & 0.48 & -21.22 & 1.76 & $\mathrm{E}$ & [B] & 5 \\
\hline 113577 & $14: 19: 56.4$ & $52: 58: 21$ & 0.67 & -22.05 & 1.56 & PD & {$[\mathrm{A}]$} & 5 \\
\hline 114966 & $14: 20: 25.1$ & $52: 50: 53$ & 0.61 & -22.18 & 1.69 & PD & $2 \mathrm{~T}, \mathrm{~S}$ & 2 \\
\hline 115327 & $14: 19: 37.2$ & 53:00:20 & 0.35 & -20.96 & 1.76 & $\mathrm{E}$ & $2 \mathrm{~F},[\mathrm{~T}]$ & 2 \\
\hline 115594 & $14: 20: 27.8$ & $52: 49: 36$ & 0.31 & -20.81 & 1.78 & $\mathrm{E}$ & $2 \mathrm{~N}, \mathrm{~T}$ & 2,3 \\
\hline 118942 & $14: 20: 21.8$ & $52: 47: 15$ & 0.37 & -21.04 & 1.63 & $\mathrm{E}$ & $\ldots$ & 5 \\
\hline 119696 & $14: 20: 18.2$ & $52: 47: 12$ & 0.50 & -21.77 & 1.68 & $\mathrm{E}$ & $\mathrm{B}, \mathrm{F}$ & 1,2 \\
\hline 122098 & $14: 19: 26.6$ & $52: 55: 17$ & 0.22 & -21.34 & 1.65 & PD & $\ldots$ & 5 \\
\hline 124509 & $14: 19: 29.8$ & $52: 51: 59$ & 0.34 & -20.75 & 1.84 & PD & $\mathrm{B}, 2 \mathrm{~T}, \mathrm{~F}$ & 1,2 \\
\hline 125663 & $14: 19: 34.8$ & $52: 49: 47$ & 0.53 & -21.53 & 1.59 & $\mathrm{E}$ & {$[\mathrm{F}]$} & 5 \\
\hline 126918 & $14: 18: 57.1$ & $52: 56: 12$ & 0.49 & -21.22 & 1.77 & $\mathrm{E}$ & $\mathrm{F},[\mathrm{B}]$ & 1,2 \\
\hline 127241 & $14: 20: 00.0$ & $52: 42: 54$ & 0.59 & -21.75 & 1.67 & $\mathrm{PD}$ & $\ldots$ & 5 \\
\hline 127457 & $14: 18: 47.0$ & $52: 57: 40$ & 0.50 & -22.08 & 1.63 & $\mathrm{E}$ & $2 \mathrm{~N}, \mathrm{~A}$ & 2,3 \\
\hline 128074 & $14: 19: 09.6$ & $52: 52: 25$ & 0.34 & -20.73 & 1.65 & $\mathrm{E}$ & $\mathrm{B},[\mathrm{F}]$ & 1 \\
\hline 128416 & $14: 19: 58.3$ & $52: 42: 01$ & 0.58 & -21.51 & 1.68 & PD & $\ldots$ & 5 \\
\hline 132682 & $14: 18: 39.8$ & $52: 53: 49$ & 0.33 & -20.83 & 1.51 & $\mathrm{E}$ & $\ldots$ & 5 \\
\hline 135859 & $14: 18: 49.0$ & $52: 48: 38$ & 0.40 & -20.88 & 1.80 & $\mathrm{E}$ & {$[\mathrm{I}]$} & 5 \\
\hline 138794 & $14: 19: 17.5$ & $52: 39: 40$ & 0.50 & -21.23 & 1.70 & $\mathrm{E}$ & {$[\mathrm{T}]$} & 5 \\
\hline 139190 & $14: 19: 00.2$ & $52: 42: 49$ & 0.44 & -20.66 & 1.66 & $\mathrm{E}$ & $\ldots$ & 5 \\
\hline 140456 & $14: 19: 08.2$ & $52: 39: 53$ & 0.30 & -21.13 & 1.78 & PD & $2 \mathrm{~T}$ & 2 \\
\hline 140758 & $14: 19: 15.4$ & $52: 38: 05$ & 0.43 & -21.31 & 1.68 & $\mathrm{E}$ & $\mathrm{S}$ & 2 \\
\hline
\end{tabular}


Table 2 - continued

\begin{tabular}{|c|c|c|c|c|c|c|c|c|}
\hline IRAC ID & RA & Dec. & $z_{\text {phot }}$ & $M_{B}$ & $(\mathrm{M} u-\mathrm{M} g)$ & Type & Morphology & Group \\
\hline 141714 & $14: 18: 47.3$ & $52: 42: 51$ & 0.44 & -20.65 & 1.76 & PD & {$[\mathrm{B}],[\mathrm{S}]$} & 5 \\
\hline 143149 & $14: 18: 07.9$ & $52: 49: 24$ & 0.37 & -21.81 & 1.77 & $\mathrm{E}$ & $\mathrm{T}$ & 2 \\
\hline 143536 & $14: 18: 33.1$ & $52: 43: 52$ & 0.50 & -21.20 & 1.81 & PD & {$[\mathrm{T}]$} & 5 \\
\hline 145098 & $14: 18: 10.6$ & $52: 46: 50$ & 0.32 & -20.84 & 1.70 & $\mathrm{E}$ & $\mathrm{A}, \mathrm{T}$ & 2 \\
\hline 145434 & $14: 18: 53.3$ & $52: 37: 43$ & 0.48 & -21.68 & 1.68 & PD & $4 \mathrm{~T}$ & 2 \\
\hline 146298 & $14: 18: 35.0$ & $52: 40: 34$ & 0.59 & -21.57 & 1.76 & $\mathrm{E}$ & {$[\mathrm{A}]$} & 5 \\
\hline 152722 & $14: 17: 41.3$ & $52: 44: 45$ & 0.49 & -20.77 & 1.55 & $\mathrm{PD}$ & {$[\mathrm{F}]$} & 5 \\
\hline 156161 & $14: 18: 36.5$ & $52: 29: 35$ & 0.30 & -20.71 & 1.79 & $\mathrm{E}$ & $\mathrm{T}$ & 2 \\
\hline 157751 & $14: 18: 24.5$ & $52: 30: 24$ & 0.47 & -21.22 & 1.81 & $\mathrm{E}$ & $\ldots$ & 5 \\
\hline 157878 & $14: 17: 30.2$ & $52: 41: 20$ & 0.46 & -20.85 & 1.70 & $\mathrm{E}$ & F & 2 \\
\hline 159123 & $14: 18: 15.8$ & $52: 30: 37$ & 0.56 & -22.03 & 1.70 & PD & $\mathrm{T}$ & 2 \\
\hline 159936 & $14: 17: 28.3$ & $52: 39: 26$ & 0.41 & -21.22 & 1.55 & $\mathrm{E}$ & $2 \mathrm{~N}$ & 3 \\
\hline 160442 & $14: 17: 33.8$ & $52: 37: 46$ & 0.47 & -21.90 & 1.61 & $\mathrm{E}$ & $\mathrm{B}, \mathrm{A}$ & 1,2 \\
\hline 160500 & $14: 17: 33.1$ & $52: 37: 53$ & 0.34 & -20.81 & 1.78 & PD & $\mathrm{B}, 2 \mathrm{~T}$ & 1,2 \\
\hline 161724 & $14: 17: 25.4$ & $52: 38: 08$ & 0.34 & -20.71 & 1.95 & PD & {$[\mathrm{F}]$} & 5 \\
\hline 165265 & $14: 17: 11.0$ & $52: 37: 29$ & 0.67 & -22.04 & 1.55 & $\mathrm{E}$ & $\mathrm{B}, \mathrm{T}$ & 1,2 \\
\hline 166730 & $14: 17: 53.5$ & $52: 27: 22$ & 0.36 & -21.01 & 1.55 & $\mathrm{E}$ & $\mathrm{S}, \mathrm{T}$ & 2 \\
\hline 169386 & $14: 16: 58.6$ & $52: 35: 49$ & 0.47 & -20.68 & 1.55 & PD & $\ldots$ & 5 \\
\hline 172474 & $14: 17: 19.9$ & $52: 28: 24$ & 0.51 & -20.90 & 1.82 & $\mathrm{E}$ & $\mathrm{T}, \mathrm{B}, \mathrm{F}$ & 1,2 \\
\hline 173901 & $14: 17: 32.2$ & $52: 24: 15$ & 0.32 & -21.39 & 1.76 & $\mathrm{E}$ & $\ldots$ & 5 \\
\hline 175347 & $14: 17: 08.9$ & $52: 27: 09$ & 0.60 & -21.92 & 1.60 & $\mathrm{E}$ & $\mathrm{S},[\mathrm{B}]$ & 2 \\
\hline 175590 & $14: 17: 15.4$ & $52: 25: 33$ & 0.56 & -21.68 & 1.71 & $\mathrm{E}$ & [A] & 5 \\
\hline 177990 & $14: 16: 41.3$ & $52: 29: 02$ & 0.25 & -21.05 & 1.75 & PD & $\mathrm{F},[2 \mathrm{~N}]$ & 2 \\
\hline 178118 & $14: 17: 20.2$ & $52: 20: 51$ & 0.46 & -21.74 & 1.75 & $\mathrm{E}$ & $\ldots$ & 5 \\
\hline 178724 & $14: 17: 15.1$ & $52: 20: 51$ & 0.52 & -21.81 & 1.67 & $\mathrm{E}$ & A & 2 \\
\hline 178868 & $14: 16: 57.5$ & $52: 24: 09$ & 0.37 & -22.14 & 1.69 & PD & F & 2 \\
\hline 180420 & $14: 16: 54.0$ & $52: 21: 50$ & 0.54 & -21.91 & 1.62 & $\mathrm{E}$ & $2 \mathrm{~N}, 2 \mathrm{~T},[\mathrm{~B}]$ & 2,3 \\
\hline 181402 & $14: 16: 38.2$ & $52: 23: 08$ & 0.38 & -20.87 & 1.76 & $\mathrm{E}$ & {$[\mathrm{I}],[\mathrm{A}]$} & 5 \\
\hline 181444 & $14: 16: 47.3$ & $52: 21: 11$ & 0.31 & -21.63 & 1.71 & $\mathrm{E}$ & $2 \mathrm{~S},[\mathrm{I}]$ & 2 \\
\hline 181736 & $14: 16: 27.4$ & $52: 24: 39$ & 0.46 & -20.93 & 1.79 & PD & $\ldots$ & 5 \\
\hline 181914 & $14: 16: 57.6$ & $52: 18: 10$ & 0.36 & -20.72 & 1.66 & PD & $\ldots$ & 5 \\
\hline 182762 & $14: 16: 52.8$ & $52: 17: 28$ & 0.43 & -21.41 & 1.71 & $\mathrm{PD}$ & {$[\mathrm{F}]$} & 5 \\
\hline 183081 & $14: 16: 49.0$ & $52: 17: 38$ & 0.36 & -21.86 & 1.71 & $\mathrm{E}$ & $\mathrm{F},[\mathrm{T}]$ & 2 \\
\hline 183836 & $14: 16: 43.2$ & $52: 17: 21$ & 0.44 & -21.02 & 1.76 & $\mathrm{E}$ & {$[\mathrm{S}]$} & 5 \\
\hline 184041 & $14: 16: 40.0$ & $52: 17: 35$ & 0.53 & -22.13 & 1.75 & $\mathrm{E}$ & $\mathrm{F}, \mathrm{S}$ & 2 \\
\hline 184315 & $14: 16: 16.8$ & $52: 21: 46$ & 0.50 & -21.61 & 1.64 & PD & $2 \mathrm{~N}$ & 3 \\
\hline 186058 & $14: 16: 08.9$ & $52: 19: 59$ & 0.54 & -21.77 & 1.80 & PD & {$[\mathrm{A}]$} & 5 \\
\hline 189727 & $14: 16: 15.1$ & $52: 11: 21$ & 0.64 & -21.96 & 1.80 & PD & $\ldots$ & 5 \\
\hline 190795 & $14: 16: 10.3$ & $52: 10: 12$ & 0.51 & -21.19 & 1.70 & PD & $\mathrm{T}, \mathrm{S}$ & 2 \\
\hline 193464 & $14: 15: 36.5$ & $52: 11: 41$ & 0.42 & -20.69 & 1.66 & $\mathrm{E}$ & $2 \mathrm{~N}, \mathrm{~F}$ & 2,3 \\
\hline 193507 & $14: 16: 03.1$ & $52: 06: 11$ & 0.47 & -21.91 & 1.71 & $\mathrm{E}$ & $2 \mathrm{~N},[\mathrm{~B}]$ & 3 \\
\hline 193737 & $14: 15: 54.5$ & $52: 07: 30$ & 0.50 & -20.88 & 1.55 & $\mathrm{E}$ & $\ldots$ & 5 \\
\hline 193974 & $14: 15: 31.4$ & $52: 11: 46$ & 0.40 & -21.20 & 1.73 & $\mathrm{E}$ & {$[\mathrm{S}]$} & 5 \\
\hline 194092 & $14: 15: 29.0$ & $52: 12: 00$ & 0.51 & -20.88 & 1.66 & $\mathrm{E}$ & {$[\mathrm{T}]$} & 5 \\
\hline 196827 & $14: 15: 41.3$ & $52: 03: 43$ & 0.37 & -20.86 & 1.76 & $\mathrm{E}$ & $\mathrm{T}$ & 2 \\
\hline 198295 & $14: 14: 58.6$ & $52: 09: 25$ & 0.54 & -21.91 & 1.72 & $\mathrm{E}$ & {$[\mathrm{S}]$} & 5 \\
\hline 199503 & $14: 14: 56.2$ & $52: 07: 26$ & 0.50 & -21.20 & 1.70 & PD & $\mathrm{T}$ & 2 \\
\hline 202111 & $14: 14: 41.3$ & $52: 04: 54$ & 0.27 & -21.16 & 1.70 & $\mathrm{E}$ & {$[\mathrm{S}]$} & 5 \\
\hline 204161 & $14: 14: 57.8$ & $51: 57: 54$ & 0.62 & -21.78 & 1.88 & $\mathrm{E}$ & $\mathrm{A},[\mathrm{B}]$ & 2 \\
\hline 204944 & $14: 14: 41.3$ & $51: 59: 40$ & 0.28 & -21.55 & 1.66 & PD & $\mathrm{T}, \mathrm{S}$ & 2 \\
\hline
\end{tabular}

of the PRGs. In Fig. 4 we present six examples of Suprime-Cam images of galaxies in the EGS, showing different levels of disturbance in their morphologies.

\section{RESULTS}

\subsection{Optical morphologies}

The main aim of this work is to perform a morphological classification of the galaxies in the OBEY and EGS samples in the same manner as for the PRGs in the 2 Jy sample studied in RA11 and then to compare the results. This comparison will allow us to determine whether or not galaxy interactions are more common in powerful AGN than in quiescent galaxies and, consequently, to establish how important these interactions are in the triggering of nuclear activity.

\subsubsection{Morphological features}

The morphological classification of the galaxies was done blind, with no information about any previous work on the sources, by CRA visually inspecting the 55 OBEY survey and the 107 EGS images. In addition, PSB and CNT also examined the EGS galaxy 

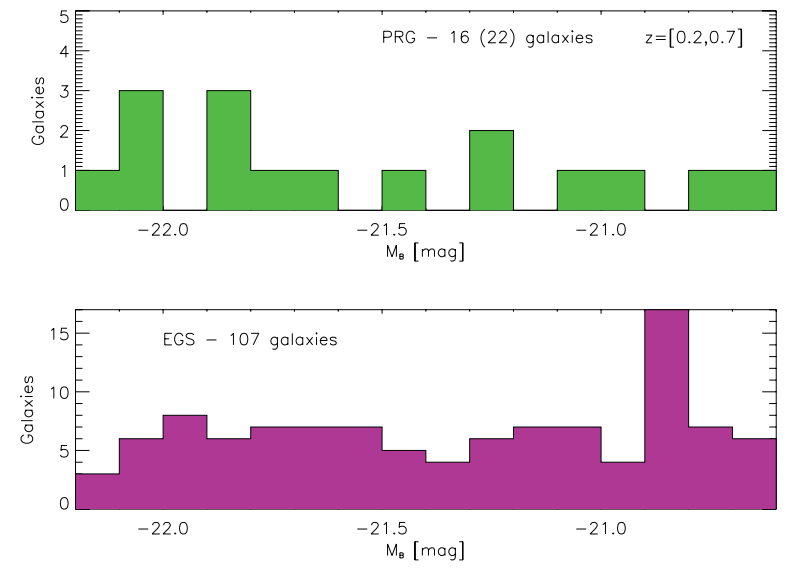

Figure 2. Comparison between the $B$-band absolute magnitudes of the PRGs in the 2 Jy sample at $0.2 \leq z<0.7$ (top panel) and those of the red galaxies in the EGS (bottom panel).
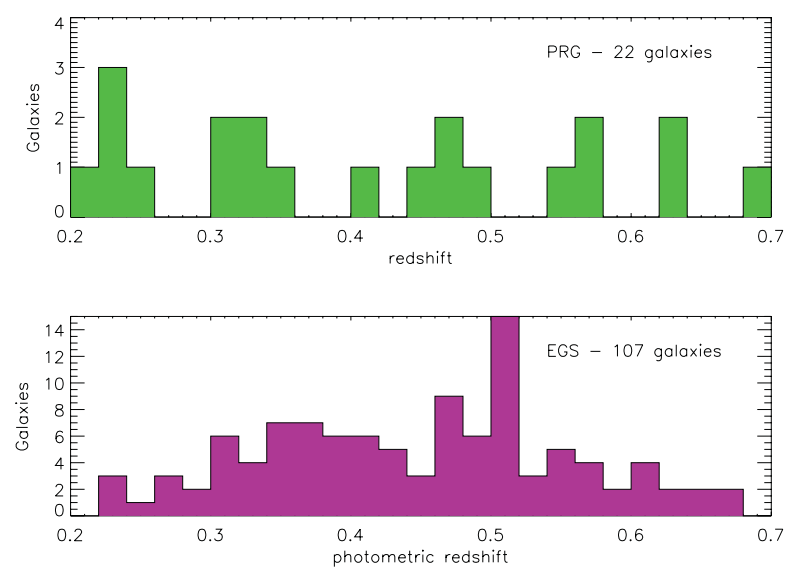

Figure 3. Comparison between the spectroscopic redshifts of the PRGs in the 2 Jy sample at and the photometric redshifts of the red galaxies in the EGS (bottom panel).

morphologies and there was agreement among the classifiers for the majority of the galaxies. Any possible conflicts were resolved by re-examining the images. The classification of the various features detected in the two control samples is based on that first used by Heckman et al. (1986) and is exactly the same as employed in RA11. Note that the classification of the PRGs was done using the fully reduced GMOS-S original images, before any image enhancement technique was applied to them. The following morphological features are considered.

(i) A tail (T) corresponds to a narrow curvilinear feature with roughly radial orientation.

(ii) A $\operatorname{fan}(\mathrm{F})$ is similar to a tail, but shorter and broader.

(iii) A bridge (B) is a feature that links the radio galaxy with a companion.

(iv) A shell (S) is a curving filamentary structure with a roughly tangential orientation to the main body of the galaxy.

(v) Dust (D) includes both nuclear dusty features and large-scale dust lanes.

(vi) Amorphous haloes (A) are irregular galaxy hosts or inner features that cannot be clearly distinguished from the main body of the galaxy (e.g. knotty haloes).

(vii) By irregular (I) we refer to any feature, generally elongated, that cannot be classified as any of the previous. (viii) Double nuclei $(2 \mathrm{~N})$ are those composed of two bright peaks inside $10 \mathrm{kpc}$, following the definition employed by Smith \& Heckman (1989), based on statistical studies of cluster galaxies (Hoessel 1980) ${ }^{5}$ and $N$-body simulations of interacting binary galaxies (Borne 1984).

All of these features, with the possible exception of the dust, are very likely the result of galaxy interactions. Simulations have shown how spiral-spiral (SS), elliptical-spiral (ES) and ellipticalelliptical (EE) interactions can produce all of the features that form the basis of our classification (Quinn 1984; Hernquist \& Spergel 1992; Cattaneo et al. 2005; Lotz et al. 2008; Feldmann, Mayer \& Carollo 2008). For a more detailed description of how the different features described above are produced, according to simulations, see section 5.1.1 in RA11. The classified features for both the OBEY survey and the EGS sample are listed in Tables 1 and 2, respectively. Features with uncertain identification (between brackets in Tables 1 and 2) have not been considered for the statistics. Examples of the EGS galaxy morphologies are shown in Fig. 4 and all the sample images can be individually viewed online in the Rainbow data base. ${ }^{6}$ For the images of the OBEY survey we refer the reader to Tal et al. (2009).

The projected linear scales of the tidal features reported in $\mathrm{Ta}-$ bles 1 and 2 range from less than $10 \mathrm{kpc}$ in the case of galaxies with double nuclei to $\sim 80-85 \mathrm{kpc}$ in the case of long tidal tails such as that in NGC 1209 (OBEY survey) and bridges linking galaxies as in the case of irac128074 (EGS sample). For the PRGs in the 2 Jy sample the longest feature that we measured was the spectacular bridge in PKS 0349-27, which links the radio galaxy with a distorted companion at $\sim 83 \mathrm{kpc}$ ( RA11).

\subsubsection{Quantitative versus visual analysis of tidal disturbance}

In Section 3.1.1 we described how we performed the visual classification of the optical morphologies of the PRG, OBEY and EGS samples. In the following, we compare the results of this visual classification of the galaxies in the OBEY survey with the quantitative analysis of the degree of tidal disturbance carried out by Tal et al. (2009) for the same galaxies. The latter authors fitted an elliptical galaxy model to the targets and, after masking the foreground stars and background galaxies in the sky-subtracted and flat-fielded images, they divided the masked frames by the galaxy model. This process produces an image of the residuals that they translated into a number, the tidal parameter, defined as: $T=\overline{\left|\left(I_{x, y} / M_{x, y}\right)-1\right|}$, where $I_{x, y}$ and $M_{x, y}$ are the pixel values at $(x, y)$ of the galaxy and model images, respectively. They finally applied a correction for the residual noise to the latter values, to derive the corrected tidal parameter $\left(T_{\mathrm{c}}\right.$; see Tal et al. 2009 for a more detailed description of the methodology).

Based on the values of $T_{\mathrm{c}}$ determined for the galaxies in the OBEY survey, Tal et al. (2009) divided them into three groups: (1) galaxies showing clear signs of morphological disturbance ( $T_{\mathrm{c}}>0.09 ; 53$ per cent of the sample), (2) galaxies with marginal disturbance $\left(0.07<T_{\mathrm{c}}<0.09 ; 20\right.$ per cent), and (3) galaxies lacking interaction signatures $\left(T_{\mathrm{c}}<0.07 ; 27\right.$ per cent). In Fig. 5 we represent these tidal parameters versus the $B$-band absolute magnitudes (see also Table 1). First, we note that there is no clear correlation

\footnotetext{
${ }^{5}$ Hoessel (1980) claimed that typical cluster members are expected to experience a close encounter or merger within this radius every $10^{9}$ years.

${ }^{6} \mathrm{https}: / /$ rainbowx.fis.ucm.es/Rainbow_navigator_public/
} 


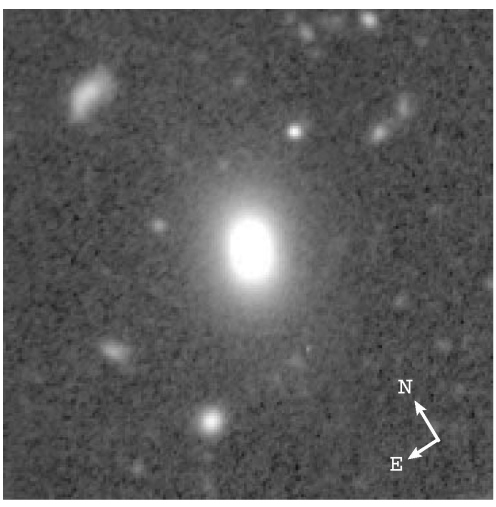

(a)

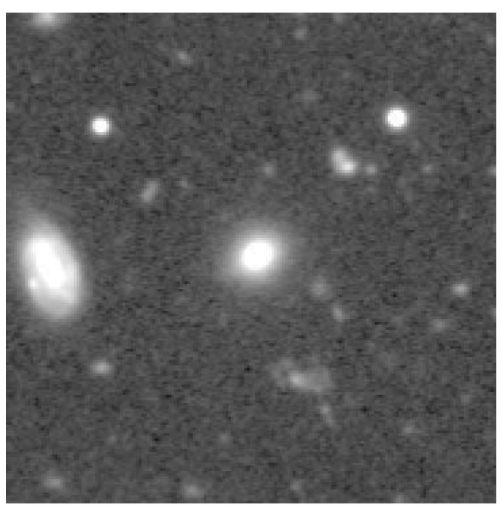

(d)

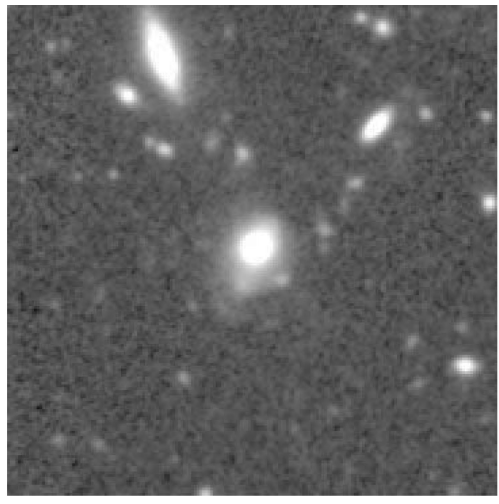

(b)

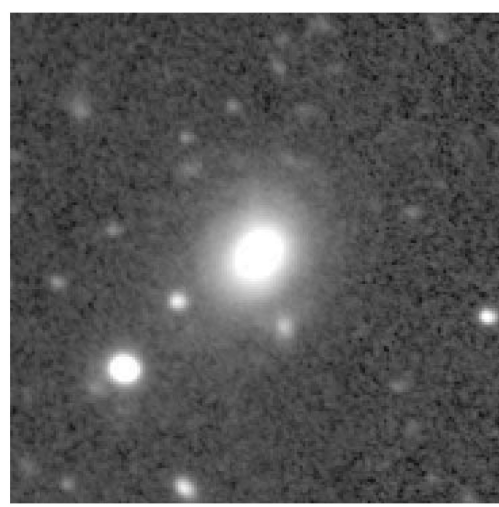

(e)

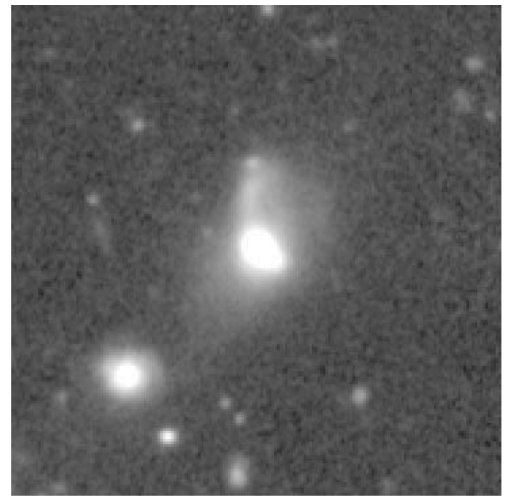

(c)

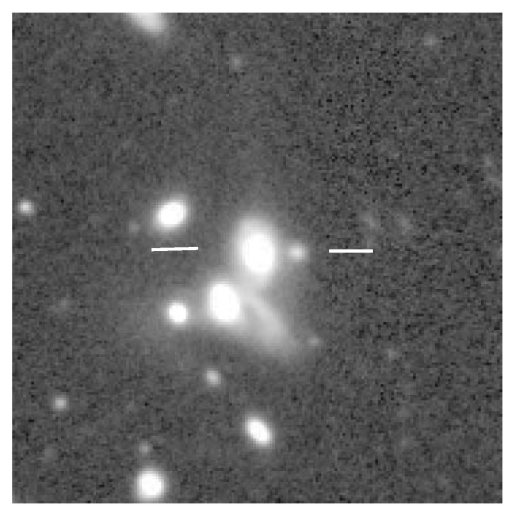

(f)

Figure 4. Examples of ET galaxies in the EGS sample. The level of disturbance in their morphologies increases from left to right. Images' size is 36 arcsec side. IDs and classification from Table 2 follow: (a) irac105193 - undisturbed; (b) irac145098 - A,T; (c) irac111427 - 2N,T,2I; (d) irac161724 - undisturbed; (e) irac204944 - T,S and (f) irac124509 - B,2T,F.

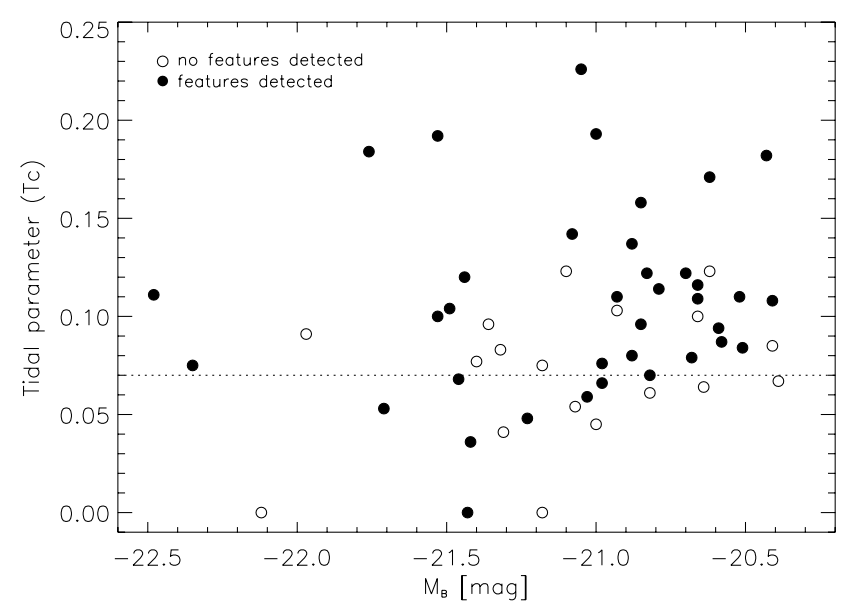

Figure 5. Corrected tidal parameter values from Tal et al. (2009) for the OBEY survey versus $B$-band absolute magnitudes. The dotted horizontal line at $T_{\mathrm{c}}=0.07$ is the boundary between galaxies with and without signs of disturbance from Tal et al. (2009). Filled circles correspond to the galaxies visually classified as disturbed in this work, whereas open circles indicate absence of morphological features.

between the luminosities of the galaxies and the level of disturbance of their hosts. Secondly, we have identified with filled circles the galaxies in the OBEY survey for which we have found signs of interaction based on our visual classification. From Fig. 5 it is clear that quantitative and visual classifications do not agree completely. There is good agreement only for the nine galaxies with
$T_{\mathrm{c}}>0.13$. In fact, for five galaxies that Tal et al. (2009) classified as clearly disturbed $\left(T_{\mathrm{c}}>0.09\right)$ we do not find any sign of interaction. After inspecting the images of these five galaxies we conclude that it is likely that the high value of $T_{\mathrm{c}}$ is due to residuals from the masking of the foreground stars and background galaxies and/or from isophotal twisting. The opposite is also true: we find disturbed morphologies in seven galaxies with $T_{\mathrm{c}}<0.07$, which are either faint relative to their host galaxies or diffuse features that the automatic method misses. Overall, however, the total percentages of disturbance agree well between the automatic ( 73 per cent) and the visual classification (67 per cent). This vindicates the use of visual rather than quantitative detection of disturbed morphologies in this paper.

\subsubsection{Classification}

Considering the morphological features detected in the OBEY and EGS galaxies (only those with secure identifications in Tables 1 and 2), the sample can be divided into the following five groups.

(1) Galaxy pair or group in tidal interaction. Galaxy pairs showing bridges, or co-aligned distorted structures.

(2) Galaxies showing tidal features. Galaxies showing shells, fans, tails, amorphous haloes and irregular features.

(3) Multiple nuclei. Galaxies with a companion lying inside a $10 \mathrm{kpc}$ radius, according to the theoretical definition employed by Hoessel (1980) and Smith \& Heckman (1989).

(4) Dust features. Galaxies presenting dust features as the only sign of disturbance. 
Table 3. Classification of all the galaxies in the PRG, OBEY and EGS samples. Sources belonging to groups 1 and 3 are considered as pre-coalescence systems, those in group 2 are likely coalescence or post-coalescence scenarios, and finally, galaxies classified in groups 4 and 5 do not show signs of interaction. * Those galaxies classified as $(2,3)$ or $(1,2)$ in Tables 1 and 2 , and table 1 in RA11 are considered as pre-coalescence systems here, although they belong to group 2 as well. Percentages between parentheses correspond to SLRGs in the PRG sample (Columns 3 and 5) and to elliptical galaxies only in the EGS sample (Column 6).

\begin{tabular}{lccccc}
\hline Morphology & Group & $\begin{array}{c}\text { PRG sample } z<0.2 \\
\text { (per cent) }\end{array}$ & $\begin{array}{c}\text { OBEY survey } \\
\text { (per cent) }\end{array}$ & $\begin{array}{c}\text { PRG sample } 0.2 \leq z<0.7 \\
\text { (per cent) }\end{array}$ & $\begin{array}{c}\text { EGS sample } \\
\text { (per cent) }\end{array}$ \\
\hline Signs of interaction & $1,2,3$ & $62(93)$ & 67 & $95(95)$ & $55(57)$ \\
& 1,3 & $21(21)$ & 7 & $50(50)$ & $20(24)$ \\
Pre-coalescence & $2^{*}$ & $42(72)$ & 60 & $45(45)$ & $35(33)$ \\
Coalescence or post-coalescence & 4,5 & $37(7)$ & 33 & $5(5)$ & $45(43)$ \\
No signs of interaction & & & & & \\
\hline
\end{tabular}

(5) Isolated galaxies with no sign of interaction. Objects in which we cannot confidently identify morphological peculiarities.

Note that these categories are not exclusive because some galaxies show more than one of the morphological features described above (see Table 1). Initially we considered objects in groups 1, 2, 3 and 4 as showing disturbed morphologies consistent with them having been involved in a galaxy interaction/merger, whilst galaxies classified in the fifth group were classified as undisturbed. Based on this classification, in RA11 we found that 85 percent of the PRG sample are very likely interacting objects or the result of a past merger event. However, dust features by themselves may not necessarily be a sign of galaxy interactions. Note that while dust is often taken as an observational signature for recent mergers (e.g. van Dokkum \& Franx 1995; Kaviraj et al. 2011), it may also be associated with cooling flows in central cluster galaxies (e.g. Fabian, Johnstone \& Daines 1994; Hansen, Jorgensen \& Norgaard-Nielsen 1995; Edge et al. 1999, 2010). If we do not consider dust as a sign of morphological disturbance related to mergers and interactions, then the percentage of PRGs in the $2 \mathrm{Jy}$ sample presenting evidence for interactions is 78 percent. In Table 3 we show the percentages of interacting galaxies (those classified in groups 1, 2 or 3) for (i) the PRGs sample at $z<0.2$ and the OBEY survey, and (ii) the PRGs sample at $0.2 \leq z<0.7$ and the EGS sample.

Thus, if we only consider the galaxies classified in groups 1,2 and 3, we find that 62 per cent of the PRGs at $z<0.2$ show signs of interactions. However, it is worth considering the percentage of disturbance after excluding the WLRGs (shown between parentheses in Table 3). As explained in the Introduction, it has been proposed that WLRGs are powered by hot gas accretion from their X-ray coronae, rather than by the classical AGN cold gas accretion (see also section 5.2.2 in RA11). Thus, if we consider SLRGs only, the percentage of PRGs showing signs of interactions increases to 93 percent, which is higher than the 67 percent that we measure for the elliptical galaxies in the OBEY survey. On the other hand, 95 per cent of the PRGs at $0.2 \leq z<0.7$ (either including or excluding the only WLRG in this redshift range) show signs of interaction, compared with 55 per cent in the EGS sample (57 per cent if we consider only ellipticals and not the PDs; Column 7 in Table 2). Thus, there appears to be more evidence for galaxy interactions in the PRG sample than in the control samples at all redshifts, although the relatively small size of the PRG sample means that the difference is only significant at the $\sim 2 \sigma$ level (for the comparison between the $0.2 \leq z<0.7$ PRG and EGS samples). However, it is necessary to consider the surface brightnesses of the features in order to make a proper comparison with the control sample morphologies, since we know that, for example, the OBEY images reach much lower effective surface brightnesses than our GMOS-S images of the PRGs. This comparison provides firmer evidence for differences between the PRG and quiescent elliptical galaxy samples, and it is discussed in Section 3.1.4.

In terms of the merger scenario, we appear to be observing some of the galaxies in the PRG and the two control samples before the final coalescence of the nuclei of the interacting system. The galaxies classified in groups 1 (galaxy pair or group in tidal interaction) and 3 (multiple nuclei) would correspond to systems observed before the coalescence of the merging nuclei, whereas those in group 2 (galaxies presenting any sign of disturbance) would correspond to more evolved systems (coalescence or post-coalescence). The percentage of galaxies in the PRG, OBEY and EGS samples belonging to each of these groups is shown in Table 3.

In RA11 we showed that, if PRGs are triggered as a consequence of an interaction between galaxies, it is possible for this to happen at any stage of the interaction (on the basis of young stellar population properties). In fact, we found that more than one-third of the PRGs in the full 2 Jy sample are observed in a pre-coalescence phase (galaxies classified in either group 1 or 3 ). Interestingly, for both the lowand intermediate-redshift subsamples, we find that the proportion of PRGs that are in the pre-coalescence phase is a factor of 2-3 higher than that of the quiescent elliptical galaxies in a similar phase (see Table 3). There is also evidence for an increase in the proportion of pre-coalescence systems with redshift for both the PRG and quiescent samples (see Table 3). However, observations of larger samples will be required to put those trends on a more solid statistical footing.

The lack of dust features in the control sample as compared to the PRG sample is also interesting. For the OBEY survey we find that only 7 per cent of the elliptical galaxies show dust, whereas for the PRGs at $z<0.2$ the percentage increases to 25 percent (21 per cent for the SLRGs). This agrees with the $\sim 30$ percent of dust features found from optical HST images of radio galaxies at $z<0.5$ (de Koff et al. 1996, 2000). Dust can either be produced by mass-loss from evolving red giant stars (Knapp et al. 1989; Athey et al. 2002) or be accreted during galaxy interactions (Goudfrooij et al. 1994; van Dokkum \& Franx 1995). If the dust is accreted in mergers/interactions, then we expect to find a higher incidence of dust features in the PRGs than in the quiescent ellipticals, mirroring the difference found for signs of disturbance in general between the two populations (see Table 7). On the other hand, at higher redshifts neither the galaxies in the EGS nor in the SLRG sample at $0.2 \leq z<0.7$ show dust features. This apparent lack of dust at higher redshifts in both the radio and the quiescent 
galaxies is likely to be due to a resolution effect, since dust lanes, with typical scales of $\sim 1-5 \mathrm{kpc}$, will be poorly resolved in the ground-based data employed here and in RA11 for galaxies at such redshifts.

We have classified the galaxies in the OBEY and EGS samples in the same manner as the PRGs and compared them. However, as we mentioned in Section 2.1, from the comparison between the $M_{B}$ histograms of the PRGs at $z<0.2$ and the OBEY sample shown in Fig. 1 , there is only a 4 per cent chance that the two distributions are drawn from the same parent population according to the KS test. In order to confirm that this difference does not produce any bias in the statistics of morphologically disturbed systems, we have split the OBEY sample into two absolute magnitude bins using the median value of $M_{B}=-20.98 \mathrm{mag}$. We find 59 percent of disturbance (filled circles in Fig. 5) in the subsample with $M_{B}<-20.98$ mag (27 galaxies) and 75 per cent if we consider $M_{B} \geq-20.98$ mag (28 galaxies). Thus, we find a slightly larger percentage of disturbed morphologies for the lower luminosity ellipticals. The galaxies in the OBEY survey are less luminous than the PRGs on average (see Fig. 1), and thus, any bias caused by this effect would result in an enhancement of the number of disturbed morphologies in the OBEY sample relative to the PRGs at $z<0.2$. However, by looking at Fig. 5 it is clear that there is no correlation between $M_{B}$ and the level of disturbance of the galaxies in the OBEY sample. Although for the galaxies in the EGS sample the $M_{B}$ distribution is more similar to that of the PRGs at $0.2 \leq z<0.7$ (0.18 of significance according to the KS test; see Section 2.2), we have performed the same test as for the ellipticals in the OBEY survey. By splitting the EGS sample into galaxies brighter and fainter than $M_{B}=-21.25$ mag (i.e. the median value), we find 60 per cent and 50 per cent of disturbance, respectively ( 53 and 54 galaxies included in each bin, respectively). Thus, we do not find any significant correlation between the levels of morphological disturbance and the luminosity of the elliptical galaxies in either the EGS or OBEY samples.

\subsubsection{Surface brightnesses}

The main result of RA11 is that 85 percent ( 78 percent if we do not consider galaxies with dust features only) of the 2 Jy sample of PRGs show peculiar optical morphologies at relatively high levels of surface brightness: (1) $\tilde{\mu}_{V}=24.0 \mathrm{mag} \operatorname{arcsec}^{-2}$ and $\Delta \mu_{V}=[22.1$, 26.2] mag $\operatorname{arcsec}^{-2}$ at $z<0.2$; and (2) $\tilde{\mu}_{V}=23.5 \mathrm{mag} \operatorname{arcsec}^{-2}$ and $\Delta \mu_{V}=[21.3,25.1] \mathrm{mag} \operatorname{arcsec}^{-2}$ at $0.2 \leq z<0.7$.

In Tables 4 and 5 we report apparent surface brightnesses ( $\mu_{V}$ in the case of the OBEY survey and $\mu_{R_{C}}$ for the EGS sample) for all the secure detections of tails, fans, shells, bridges, amorphous haloes and irregularfeatures detected in the control sample images. These surface brightnesses have been obtained exactly in the same manner as those reported in RA11. We first calculated the averaged number of counts of each feature using small apertures, and then repeated the process, using the same aperture, in several regions of the galaxy on either side of the feature to subtract the sky and the diffuse host galaxy background. In order to test the robustness of this technique, CRA and PSB measured the surface brightnesses of the same features for some of the galaxies independently, obtaining differences below $0.1 \mathrm{mag}$.

For the OBEY survey, in Table 4 we report the $\mu_{V}^{\text {corr }}$ values, obtained after correcting $\mu_{V}$ from Galactic extinction (Schlegel, Finkbeiner \& Davis 1998). For the galaxies in the EGS sample, which are at redshifts $0.2 \leq z<0.7$, the surface brightnesses were $K$-corrected, using the values reported in Frei \& Gunn (1994) and Fukugita et al. (1995) for elliptical galaxies, in addition to the $(1+z)^{4}$ cosmological dimming and extinction corrections. ${ }^{7}$ The final $\mu_{R_{c}}^{\text {corr }}$ values for the EGS sample are shown in Table 5. We finally transform the $\mu_{R_{C}}^{\text {corr }}$ values into $V$-band measurements to compare with the OBEY sample and the PRGs by assuming colours of elliptical galaxies from Frei \& Gunn (1994) and Fukugita et al. (1995).

We have chosen $K$-corrections and colour transformations for elliptical galaxies, but some of the features may be produced in mergers involving small disc galaxies and/or there can be local star formation taking place in tidal features associated with the interaction. In such cases the galaxy colours would be more similar to those of spiral galaxies. In order to assess the importance of this effect, in RA11 we re-calculated the $\mu_{V}^{\text {corr }}$ values of the features by using $K$ corrections and colours of Sbc-type spiral galaxies and confirmed that they did not change significantly $\left(\sim 0.1 \mathrm{mag} \operatorname{arcsec}^{-2}\right)$.

The comparison between $\mu_{V}^{\text {corr }}$ values measured for the features detected in our PRGs and those for the OBEY survey is shown in Fig. 6(a) and Table 6. In Fig. 6(b) we show the same comparison, but considering only the brightest disturbed feature of each galaxy. According to the KS test, the PRG and OBEY distributions shown in Figs 6(a) and (b) are different at the 99.9 percent significance level $(>3 \sigma)$.

The median depth and range of the detected features in the OBEY galaxies are $\tilde{\mu}_{V}^{\text {corr }}=25.8 \mathrm{mag} \mathrm{arcsec}^{-2}$ and $\Delta \mu_{V}=[23.4$, 28.2] mag $\operatorname{arcsec}^{-2}$, respectively. Thus, the features detected in PRGs at $z<0.2$ are $\sim 2$ mag brighter than in their quiescent counterparts. In fact, if we only consider the features in the OBEY survey which have surface brightnesses within the range of the PRGs at $z<0.2$ (i.e. $\mu_{V}^{\text {corr }} \leq 26.2 \mathrm{mag} \mathrm{arcsec}^{-2}$, which is exactly the same if we consider SLRGs only; see Table 6), the percentage of objects with morphological disturbance goes down to 53 percent. Thus, when the same range of surface brightness is considered, the proportion of interacting systems found in the PRG sample is considerably larger ( 93 per cent if we consider SLRGs only) than that found for quiescent ellipticals.

In Fig. 7 and Table 6 we show the same comparisons, but this time for the galaxies in the EGS sample and the $0.2 \leq z<0.7$ PRGs. Note that for the galaxies with 'multiple nuclei' as the only detected feature there are no measurements of surface brightnesses and thus, they are not included in Figs 6 and 7. However, those galaxies have been considered in the statistics presented in Table 7. The differences between the two distributions in Fig. 7(a) are significant at the $2 \sigma$ level: 98 percent significance according to the KS test and 95 percent if we consider the brightest features only (Fig. 7b). We measured $\tilde{\mu}_{V}^{\text {corr }}=24.2 \mathrm{mag} \mathrm{arcsec}^{-2}$ and $\Delta \mu_{V}=[22.3,26.3] \mathrm{mag} \mathrm{arcsec}^{-2}$ for the EGS sample. We emphasize that the results obtained by including or excluding objects with PD components are exactly the same. Thus, for the sake of simplicity, in the following we will consider the whole sample of 107 galaxies (including ellipticals and PDs; see Table 1).

The surface brightnesses measured for the features of the PRGs at $0.2 \leq z<0.7$ are $\sim 1$ mag brighter than those of quiescent red galaxies of similar masses and redshifts. If we only consider the galaxies in the EGS with features within the same $\mu_{V}^{\text {corr }}$ range

\footnotetext{
${ }^{7}$ Values of $E(B-V)$ from Schlegel et al. (1998) were used for each of the four Suprime-Cam images in the EGS, together with the Cardelli, Clayton $\&$ Mathis (1989) extinction law to derive the corresponding $A_{R_{C}}$ values.
} 
Table 4. Surface brightness measurements of the detected features in the $V$ band (Vega system). Column 2 lists our morphological classification (same as in Table 1). Apparent surface brightnesses and those corrected of galactic extinction $\left(\mathrm{A}_{V}\right)$ for secure identifications of T, F, S, B, A and I are given in Columns 3 and 4, respectively. Brackets in Column 2 indicate uncertain identification.

\begin{tabular}{|c|c|c|c|}
\hline ID & Morphology & $\mu_{V}\left(\operatorname{mag} \operatorname{arcsec}^{-2}\right)$ & $\mu_{V}^{\text {corr }}\left(\operatorname{mag} \operatorname{arcsec}^{-2}\right)$ \\
\hline NGC 0584 & $2 \mathrm{~S}, \mathrm{~B}$ & $25.6,26.9,25.9$ & $25.5,26.8,25.8$ \\
\hline NGC 0596 & $\mathrm{~S}, \mathrm{~F}$ & $25.7,26.1$ & $25.6,26.0$ \\
\hline NGC 0720 & $2 \mathrm{~F}$ & $26.0,26.0$ & $25.9,25.9$ \\
\hline NGC 1199 & $\ldots$ & $\ldots$ & $\ldots$ \\
\hline NGC 1209 & $\mathrm{~T}, 3 \mathrm{~F},[\mathrm{D}]$ & $27.7,25.5,25.2,25.8$ & $27.6,25.4,25.1,25.7$ \\
\hline NGC 1399 & $\ldots$ & $\ldots$ & $\ldots$ \\
\hline NGC 1395 & $3 \mathrm{~S}$ & $25.9,26.4,26.1$ & $25.8,26.3,26.0$ \\
\hline NGC 1407 & $\ldots$ & $\ldots$ & $\ldots$ \\
\hline NGC 2865 & $3 \mathrm{~S}, 2 \mathrm{~T},[\mathrm{D}]$ & $24.6,23.7,24.7,25.8,26.0$ & $24.3,23.4,24.4,25.5,25.7$ \\
\hline NGC 2974 & $\mathrm{~S},[\mathrm{D}]$ & 23.9 & 23.7 \\
\hline NGC 2986 & {$[\mathrm{~B}]$} & $\ldots$ & $\ldots$ \\
\hline NGC 3078 & $\ldots$ & $\ldots$ & $\ldots$ \\
\hline NGC 3258 & {$[2 \mathrm{~N}]$} & $\ldots$ & $\ldots$ \\
\hline NGC 3268 & $\mathrm{~S}$ & 25.5 & 25.2 \\
\hline NGC 3557B & $2 \mathrm{I}$ & $25.1,25.0$ & $24.8,24.7$ \\
\hline NGC 3557 & $\mathrm{~F},[\mathrm{~S}]$ & 25.5 & 25.2 \\
\hline NGC 3585 & $2 S$ & $26.0,25.4$ & $25.8,25.2$ \\
\hline NGC 3640 & $\mathrm{~S}, 4 \mathrm{~F}$ & $26.5,26.1,25.5,24.9,23.9$ & $26.4,26.0,25.4,24.8,23.8$ \\
\hline NGC 3706 & $2 \mathrm{~S}$ & $23.9,25.3$ & $23.6,25.0$ \\
\hline NGC 3904 & $\mathrm{~S}$ & 25.2 & 25.0 \\
\hline NGC 3923 & $4 \mathrm{~S}$ & $25.8,25.3,24.8,24.4$ & $25.6,25.1,24.6,24.2$ \\
\hline NGC 3962 & $\mathrm{~S}$ & 25.7 & 25.6 \\
\hline NGC 4105 & $2 \mathrm{~F}, \mathrm{~T}$ & $26.1,25.7,25.3$ & $25.9,25.5,25.1$ \\
\hline NGC 4261 & $\mathrm{~T}, \mathrm{~F}$ & $26.8,26.7$ & $26.7,26.6$ \\
\hline NGC 4365 & $\mathrm{~F}$ & 25.9 & 25.8 \\
\hline IC 3370 & $\mathrm{~F}, \mathrm{~S}, \mathrm{D}$ & $25.3,25.3$ & $25.0,25.0$ \\
\hline NGC 4472 & $\ldots$ & $\ldots$ & $\ldots$ \\
\hline NGC 4636 & $\mathrm{~F}$ & 26.9 & 26.8 \\
\hline NGC 4645 & $\ldots$ & $\ldots$ & $\ldots$ \\
\hline NGC 4697 & $\ldots$ & $\ldots$ & $\ldots$ \\
\hline NGC 4696 & S,D & 26.3 & 26.0 \\
\hline NGC 4767 & $2 \mathrm{~S},[\mathrm{D}]$ & $23.7,26.0$ & $23.4,25.7$ \\
\hline NGC 5011 & $\ldots$ & $\ldots$ & $\ldots$ \\
\hline NGC 5018 & $3 \mathrm{~T}, 3 \mathrm{~S},[\mathrm{D}]$ & $25.1,25.7,27.1,24.7,26.1,24.5$ & $24.8,25.4,26.8,24.4,25.8,24.2$ \\
\hline NGC 5044 & $\ldots$ & $\ldots$ & $\ldots$ \\
\hline NGC 5061 & $\mathrm{~T}, \mathrm{~S}$ & $26.0,25.0$ & $25.8,24.8$ \\
\hline NGC 5077 & {$[\mathrm{~S}],[\mathrm{D}]$} & $\ldots$ & $\ldots$ \\
\hline NGC 5576 & $3 \mathrm{~T}, \mathrm{~S}$ & $26.7,25.3,25.8,25.9$ & $26.6,25.2,25.7,25.8$ \\
\hline NGC 5638 & $\mathrm{~T}, \mathrm{~S}$ & $28.1,28.3$ & $28.0,28.2$ \\
\hline NGC 5812 & $\mathrm{~T}$ & 27.8 & 27.5 \\
\hline NGC 5813 & $\ldots$ & $\ldots$ & $\ldots$ \\
\hline NGC 5846 & $3 \mathrm{~S}, 2 \mathrm{~N}$ & $26.7,26.6,27.0$ & $26.5,26.4,26.8$ \\
\hline NGC 5898 & $3 \mathrm{~T}, \mathrm{D}, 2 \mathrm{~N}$ & $27.3,26.5,27.6$ & $26.9,26.1,27.2$ \\
\hline NGC 5903 & $\ldots$ & $\ldots$ & $\ldots$ \\
\hline IC 4797 & $\mathrm{~T}, \mathrm{I},[\mathrm{D}]$ & $26.4,25.8$ & $26.2,25.6$ \\
\hline IC 4889 & $\mathrm{~F}$ & 26.4 & 26.2 \\
\hline NGC 6861 & $\mathrm{D}$ & $\ldots$ & $\ldots$ \\
\hline NGC 6868 & $\ldots$ & $\ldots$ & $\ldots$ \\
\hline NGC 6958 & $3 \mathrm{~S},[\mathrm{D}]$ & $26.2,26.6,28.1$ & $26.1,26.5,28.0$ \\
\hline NGC 7029 & $\ldots$ & $\ldots$ & $\ldots$ \\
\hline NGC 7144 & $\ldots$ & $\ldots$ & $\ldots$ \\
\hline NGC 7196 & $\mathrm{~S},[\mathrm{D}]$ & 27.1 & 27.0 \\
\hline NGC 7192 & S & 27.5 & 27.4 \\
\hline IC 1459 & $4 \mathrm{~S}$ & $26.8,26.6,26.1,26.5$ & $26.7,26.5,26.0,26.4$ \\
\hline NGC 7507 & $\mathrm{~S}$ & 28.1 & 28.0 \\
\hline
\end{tabular}

as the PRGs at $0.2 \leq z<0.7\left(\mu_{V}^{\text {corr }} \leq 25.1 \mathrm{mag} \mathrm{arcsec}^{-2}\right)$, the percentage of objects with disturbed morphologies is 48 percent. Again, this percentage is considerably lower than the number of interacting systems found for the PRG sample at $0.2 \leq z<$
0.7 (95 percent; see Table 7). In these statistics we have included the systems classified as 'multiple nuclei', even if it is not possible to calculate a value of $\mu_{V}^{\text {corr }}$ for this type of morphology. 
Table 5. Surface brightness measurements of the detected features in the $R_{c}$ band (AB system). Column 2 corresponds to the surface brightness dimming from NED, Column 3 indicates whether a galaxy has been visually classified as an elliptical (E) or as a PD, and Column 4 lists our morphological classification as in Table 1. Apparent and corrected (including galactic extinction, dimming and $k$-corrections) surface brightness for secure identifications of T, F, S, B, A and I are given in Columns 5 and 6, respectively. Brackets in Column 4 indicate uncertain identification.

\begin{tabular}{|c|c|c|c|c|c|}
\hline IRAC ID & $\begin{array}{c}\text { Dimming } \\
\left(\mathrm{mag} \mathrm{arcsec}^{-2}\right)\end{array}$ & Type & Morphology & $\begin{array}{l}\mu_{R_{C}} \\
\left(\mathrm{mag} \operatorname{arcsec}^{-2}\right)\end{array}$ & $\begin{array}{l}\mu_{R_{C}}^{\text {corr }} \\
\left(\mathrm{mag} \operatorname{arcsec}^{-2}\right)\end{array}$ \\
\hline 004162 & 1.7 & $\mathrm{E}$ & $\ldots$ & $\ldots$ & $\ldots$ \\
\hline 006612 & 1.2 & $\mathrm{E}$ & $\mathrm{B}, \mathrm{F},[\mathrm{D}],[\mathrm{T}]$ & $23.5,24.2$ & $21.9,22.6$ \\
\hline 006613 & 1.1 & $\mathrm{E}$ & B & 24.7 & 23.1 \\
\hline 056690 & 1.8 & $\mathrm{E}$ & {$[\mathrm{A}],[\mathrm{B}]$} & $\ldots$ & $\ldots$ \\
\hline 060191 & 2.0 & $\mathrm{E}$ & $\mathrm{F}$ & 25.1 & 22.1 \\
\hline 060958 & 1.5 & PD & $\mathrm{T},[\mathrm{A}],[\mathrm{B}]$ & 26.2 & 24.2 \\
\hline 061249 & 2.2 & PD & {$[\mathrm{T}]$} & $\ldots$ & $\ldots$ \\
\hline 066105 & 1.8 & $\mathrm{E}$ & {$[\mathrm{A}]$} & $\ldots$ & $\ldots$ \\
\hline 067417 & 1.4 & $\mathrm{E}$ & $\ldots$ & $\ldots$ & $\ldots$ \\
\hline 072533 & 1.2 & $\mathrm{E}$ & $\mathrm{S}$ & 25.2 & 23.5 \\
\hline 073519 & 1.7 & $\mathrm{E}$ & {$[\mathrm{A}]$} & $\ldots$ & $\ldots$ \\
\hline 074777 & 1.5 & $\mathrm{E}$ & {$[\mathrm{S}]$} & $\ldots$ & $\ldots$ \\
\hline 074924 & 1.5 & $\mathrm{E}$ & $\ldots$ & $\ldots$ & $\ldots$ \\
\hline 077695 & 1.3 & $\mathrm{PD}$ & $\mathrm{T}$ & 26.2 & 24.5 \\
\hline 079968 & 2.0 & $\mathrm{E}$ & $\mathrm{F}$ & 26.7 & 23.5 \\
\hline 082325 & 1.9 & $\mathrm{E}$ & {$[\mathrm{F}]$} & $\ldots$ & $\ldots$ \\
\hline 083714 & 1.8 & $\mathrm{E}$ & $\mathrm{F}$ & 25.8 & 23.2 \\
\hline 088031 & 1.8 & $\mathrm{E}$ & $\mathrm{F}$ & 26.8 & 24.3 \\
\hline 090430 & 1.4 & $\mathrm{E}$ & $\mathrm{A}, \mathrm{F},[\mathrm{B}]$ & $25.1,26.2$ & $23.1,24.3$ \\
\hline 092065 & 1.9 & $\mathrm{E}$ & $\mathrm{B}$ & 25.3 & 22.4 \\
\hline 092765 & 1.3 & $\mathrm{PD}$ & {$[\mathrm{A}],[\mathrm{T}]$} & $\ldots$ & $\ldots$ \\
\hline 093764 & 1.4 & $\mathrm{E}$ & {$[\mathrm{S}]$} & $\ldots$ & $\ldots$ \\
\hline 094231 & 1.5 & $\mathrm{E}$ & $2 \mathrm{~F},[\mathrm{~T}]$ & $25.0,25.2$ & $22.9,23.1$ \\
\hline 094966 & 1.6 & $\mathrm{E}$ & $2 \mathrm{~T}$ & $26.0,26.4$ & $23.7,24.1$ \\
\hline 095727 & 1.4 & $\mathrm{E}$ & $\mathrm{F}, \mathrm{S}$ & $27.2,25.7$ & $25.3,23.7$ \\
\hline 099954 & 1.0 & $\mathrm{E}$ & {$[\mathrm{T}]$} & $\ldots$ & $\ldots$ \\
\hline 102757 & 0.9 & $\mathrm{E}$ & $2 \mathrm{~S}$ & $26.1,26.2$ & $25.0,25.0$ \\
\hline 102982 & 2.0 & $\mathrm{E}$ & F & 25.0 & 21.8 \\
\hline 103198 & 1.4 & $\mathrm{E}$ & 2N,F,S & $26.5,25.4$ & $24.6,23.5$ \\
\hline 104038 & 1.6 & $\mathrm{E}$ & B & 24.4 & 22.0 \\
\hline 104729 & 2.1 & $\mathrm{PD}$ & $\mathrm{A}$ & 25.5 & 22.1 \\
\hline 105193 & 0.9 & $\mathrm{E}$ & {$[\mathrm{S}]$} & $\ldots$ & $\ldots$ \\
\hline 106324 & 1.0 & $\mathrm{E}$ & {$[\mathrm{T}]$} & $\ldots$ & $\ldots$ \\
\hline 106984 & 1.6 & $\mathrm{PD}$ & $\mathrm{A},[\mathrm{I}]$ & 26.2 & 23.9 \\
\hline 111427 & 1.2 & $\mathrm{E}$ & $2 \mathrm{~N}, \mathrm{~T}, 2 \mathrm{I}$ & $24.5,26.4,25.7$ & $22.9,24.8,24.1$ \\
\hline 112580 & 1.8 & $\mathrm{E}$ & [B] & $\ldots$ & $\ldots$ \\
\hline 113088 & 1.7 & $\mathrm{E}$ & [B] & $\ldots$ & $\ldots$ \\
\hline 113577 & 2.2 & PD & {$[\mathrm{A}]$} & $\ldots$ & $\ldots$ \\
\hline 114966 & 2.1 & PD & $2 \mathrm{~T}, \mathrm{~S}$ & $25.2,25.3,26.2$ & $21.9,22.1,22.9$ \\
\hline 115327 & 1.3 & $\mathrm{E}$ & $2 \mathrm{~F},[\mathrm{~T}]$ & $27.3,27.6$ & $25.6,25.8$ \\
\hline 115594 & 1.2 & $\mathrm{E}$ & $2 \mathrm{~N}, \mathrm{~T}$ & 26.5 & 24.9 \\
\hline 118942 & 1.4 & $\mathrm{E}$ & $\ldots$ & $\ldots$ & $\ldots$ \\
\hline 119696 & 1.8 & $\mathrm{E}$ & $\mathrm{B}, \mathrm{F}$ & $24.9,26.2$ & $22.3,23.6$ \\
\hline 122098 & 0.9 & $\mathrm{PD}$ & $\ldots$ & $\ldots$ & $\ldots$ \\
\hline 124509 & 1.3 & $\mathrm{PD}$ & $\mathrm{B}, 2 \mathrm{~T}, \mathrm{~F}$ & $24.3,24.1,27.3,27.0$ & $22.6,22.4,25.6,25.3$ \\
\hline 125663 & 1.8 & $\mathrm{E}$ & {$[\mathrm{F}]$} & $\ldots$ & $\ldots$ \\
\hline 126918 & 1.7 & $\mathrm{E}$ & $\mathrm{F},[\mathrm{B}]$ & 25.8 & 23.2 \\
\hline 127241 & 2.0 & PD & $\ldots$ & $\ldots$ & $\ldots$ \\
\hline 127457 & 1.7 & $\mathrm{E}$ & $2 \mathrm{~N}, \mathrm{~A}$ & 24.8 & 22.2 \\
\hline 128074 & 1.3 & $\mathrm{E}$ & $\mathrm{B},[\mathrm{F}]$ & 26.9 & 25.2 \\
\hline 128416 & 2.0 & $\mathrm{PD}$ & $\ldots$ & $\ldots$ & $\ldots$ \\
\hline 132682 & 1.2 & $\mathrm{E}$ & $\ldots$ & $\ldots$ & $\ldots$ \\
\hline 135859 & 1.4 & $\mathrm{E}$ & {$[\mathrm{I}]$} & $\ldots$ & $\ldots$ \\
\hline 138794 & 1.8 & $\mathrm{E}$ & {$[\mathrm{T}]$} & $\ldots$ & $\ldots$ \\
\hline 139190 & 1.6 & $\mathrm{E}$ & $\ldots$ & $\ldots$ & $\ldots$ \\
\hline 140456 & 1.1 & $\mathrm{PD}$ & $2 \mathrm{~T}$ & $24.5,25.3$ & $23.0,23.8$ \\
\hline 140758 & 1.6 & $\mathrm{E}$ & $\mathrm{S}$ & 26.0 & 23.8 \\
\hline
\end{tabular}


Table 5 - continued

\begin{tabular}{|c|c|c|c|c|c|}
\hline IRAC ID & $\begin{array}{c}\text { Dimming } \\
\left(\mathrm{mag}^{\left.-\operatorname{arcsec}^{-2}\right)}\right.\end{array}$ & Type & Morphology & $\begin{array}{l}\mu_{R_{C}} \\
\left(\operatorname{mag} \operatorname{arcsec}^{-2}\right)\end{array}$ & $\begin{array}{l}\mu_{R c}^{\text {corr }} \\
\left(\operatorname{mag}^{-2} \operatorname{arcsec}^{-2}\right)\end{array}$ \\
\hline 141714 & 1.6 & PD & {$[\mathrm{B}],[\mathrm{S}]$} & $\ldots$ & $\ldots$ \\
\hline 143149 & 1.4 & $\mathrm{E}$ & $\mathrm{T}$ & 25.8 & 23.9 \\
\hline 143536 & 1.8 & PD & {$[\mathrm{T}]$} & $\ldots$ & $\ldots$ \\
\hline 145098 & 1.2 & $\mathrm{E}$ & $\mathrm{A}, \mathrm{T}$ & $25.7,27.2$ & $24.0,25.6$ \\
\hline 145434 & 1.7 & $\mathrm{PD}$ & $4 \mathrm{~T}$ & $24.6,25.7,25.1,25.7$ & $22.2,23.2,22.6,23.2$ \\
\hline 146298 & 2.0 & $\mathrm{E}$ & {$[\mathrm{A}]$} & $\ldots$ & $\ldots$ \\
\hline 152722 & 1.7 & PD & {$[\mathrm{F}]$} & $\ldots$ & $\ldots$ \\
\hline 156161 & 1.1 & $\mathrm{E}$ & $\mathrm{T}$ & 24.5 & 23.0 \\
\hline 157751 & 1.7 & $\mathrm{E}$ & $\ldots$ & $\ldots$ & $\ldots$ \\
\hline 157878 & 1.6 & $\mathrm{E}$ & $\mathrm{F}$ & 26.3 & 24.0 \\
\hline 159123 & 1.9 & $\mathrm{PD}$ & $\mathrm{T}$ & 25.7 & 22.8 \\
\hline 159936 & 1.5 & $\mathrm{E}$ & $2 \mathrm{~N}$ & $\ldots$ & $\ldots$ \\
\hline 160442 & 1.7 & $\mathrm{E}$ & $\mathrm{B}, \mathrm{A}$ & $26.5,25.6$ & $24.1,23.2$ \\
\hline 160500 & 1.3 & PD & $\mathrm{B}, 2 \mathrm{~T}$ & $26.7,26.0,25.4$ & $24.9,24.3,23.7$ \\
\hline 161724 & 1.3 & PD & {$[\mathrm{F}]$} & $\ldots$ & $\ldots$ \\
\hline 165265 & 2.2 & $\mathrm{E}$ & $\mathrm{B}, \mathrm{T}$ & $25.7,26.2$ & $22.0,22.4$ \\
\hline 166730 & 1.3 & $\mathrm{E}$ & $\mathrm{S}, \mathrm{T}$ & $25.8,27.0$ & $23.9,25.1$ \\
\hline 169386 & 1.7 & PD & $\ldots$ & $\ldots$ & $\ldots$ \\
\hline 172474 & 1.8 & $\mathrm{E}$ & $\mathrm{T}, \mathrm{B}, \mathrm{F}$ & $26.3,27.9,26.6$ & $23.7,25.3,23.9$ \\
\hline 173901 & 1.2 & $\mathrm{E}$ & $\ldots$ & $\ldots$ & $\ldots$ \\
\hline 175347 & 2.0 & $\mathrm{E}$ & $\mathrm{S},[\mathrm{B}]$ & 26.4 & 23.2 \\
\hline 175590 & 1.9 & $\mathrm{E}$ & {$[\mathrm{A}]$} & $\ldots$ & $\ldots$ \\
\hline 177990 & 1.0 & PD & $\mathrm{F},[2 \mathrm{~N}]$ & 26.1 & 24.8 \\
\hline 178118 & 1.6 & $\mathrm{E}$ & $\ldots$ & $\ldots$ & $\ldots$ \\
\hline 178724 & 1.8 & E & A & 26.6 & 23.9 \\
\hline 178868 & 1.4 & PD & F & 26.1 & 24.2 \\
\hline 180420 & 1.9 & $\mathrm{E}$ & $2 \mathrm{~N}, 2 \mathrm{~T},[\mathrm{~B}]$ & $25.8,26.2$ & $22.9,23.4$ \\
\hline 181402 & 1.4 & E & {$[\mathrm{I}],[\mathrm{A}]$} & $\ldots$ & $\ldots$ \\
\hline 181444 & 1.2 & $\mathrm{E}$ & $2 \mathrm{~S},[\mathrm{I}]$ & $25.5,24.8$ & $23.9,23.3$ \\
\hline 181736 & 1.7 & PD & $\ldots$ & $\ldots$ & $\ldots$ \\
\hline 181914 & 1.3 & $\mathrm{PD}$ & $\ldots$ & $\ldots$ & $\ldots$ \\
\hline 182762 & 1.5 & PD & {$[\mathrm{F}]$} & $\ldots$ & $\ldots$ \\
\hline 183081 & 1.3 & $\mathrm{E}$ & $\mathrm{F},[\mathrm{T}]$ & 26.8 & 24.9 \\
\hline 183836 & 1.6 & $\mathrm{E}$ & {$[\mathrm{S}]$} & $\ldots$ & $\ldots$ \\
\hline 184041 & 1.8 & $\mathrm{E}$ & $\mathrm{F}, \mathrm{S}$ & $25.4,26.5$ & $22.6,23.7$ \\
\hline 184315 & 1.8 & PD & $2 \mathrm{~N}$ & $\ldots$ & $\ldots$ \\
\hline 186058 & 1.9 & PD & {$[\mathrm{A}]$} & $\ldots$ & $\ldots$ \\
\hline 189727 & 2.1 & PD & $\ldots$ & $\ldots$ & $\ldots$ \\
\hline 190795 & 1.8 & PD & $\mathrm{T}, \mathrm{S}$ & $25.2,26.1$ & $22.6,23.4$ \\
\hline 193464 & 1.5 & $\mathrm{E}$ & $2 \mathrm{~N}, \mathrm{~F}$ & 25.5 & 23.4 \\
\hline 193507 & 1.7 & $\mathrm{E}$ & $2 \mathrm{~N},[\mathrm{~B}]$ & $\ldots$ & $\ldots$ \\
\hline 193737 & 1.7 & E & $\ldots$ & $\ldots$ & $\ldots$ \\
\hline 193974 & 1.5 & E & {$[\mathrm{S}]$} & $\ldots$ & $\ldots$ \\
\hline 194092 & 1.8 & E & {$[\mathrm{T}]$} & $\ldots$ & $\ldots$ \\
\hline 196827 & 1.4 & $\mathrm{E}$ & $\mathrm{T}$ & 26.6 & 24.7 \\
\hline 198295 & 1.9 & $\mathrm{E}$ & {$[\mathrm{S}]$} & $\ldots$ & $\ldots$ \\
\hline 199503 & 1.7 & PD & $\mathrm{T}$ & 26.8 & 24.3 \\
\hline 202111 & 1.0 & $\mathrm{E}$ & {$[\mathrm{S}]$} & $\ldots$ & $\ldots$ \\
\hline 204161 & 2.1 & $\mathrm{E}$ & $\mathrm{A},[\mathrm{B}]$ & 26.0 & 22.6 \\
\hline 204944 & 1.1 & PD & $\mathrm{T}, \mathrm{S}$ & 26.3 & 24.9 \\
\hline
\end{tabular}

\section{DISCUSSION}

\subsection{Comparison between active and quiescent elliptical galaxies}

As explained in Section 1, if galaxy interactions are the main triggering mechanism for the radio-loud AGN activity, then we expect to find stronger and more common signs of morphological disturbance in the radio source host galaxies than in the general population of quiescent elliptical galaxies. The majority of quiescent luminous ellipticals were likely assembled through gas-poor mergers (van Dokkum 2005), whereas to trigger and feed a powerful radio source it is likely to require a larger gas supply. According to simulations, the morphological signatures of gas-rich interactions (such as tidal tails, shells, bridges, etc.) are brighter than those produced in gaspoor interactions (Naab et al. 2006; Bell et al. 2006; McIntosh et al. 2008).

We have compared the morphologies of the 2 Jy sample of radio galaxies with, first, a sample of ellipticals at redshift $z<0.2$ and, secondly, with a sample of ET galaxies at $0.2 \leq z<0.7$. We find 
Table 6. Median values and ranges of the surface brightness measurements of the PRG, OBEY and EGS samples. Values considering all the features detected (two top rows) and the brightest feature of each galaxy (bottom rows) are listed. Surface brightnesses between parentheses correspond to SLRGs in the PRG sample.

\begin{tabular}{|c|c|c|c|c|c|c|c|c|}
\hline \multirow[t]{2}{*}{ Morphology } & \multicolumn{2}{|c|}{ PRG sample $z<0.2$} & \multicolumn{2}{|c|}{ OBEY survey } & \multicolumn{2}{|c|}{ PRG sample $0.2 \leq z<0.7$} & \multicolumn{2}{|c|}{ EGS sample } \\
\hline & $\tilde{\mu}_{V}^{\text {corr }}$ & $\Delta \mu_{V}$ & $\tilde{\mu}_{V}^{\text {corr }}$ & $\Delta \mu_{V}$ & $\tilde{\mu}_{V}^{\text {corr }}$ & $\Delta \mu_{V}$ & $\tilde{\mu}_{V}^{\text {corr }}$ & $\Delta \mu_{V}$ \\
\hline All features & $\begin{array}{c}24.1 \\
(24.3)\end{array}$ & $\begin{array}{c}22.1-26.2 \\
(22.6-26.2)\end{array}$ & $\begin{array}{c}25.8 \\
\ldots\end{array}$ & $\begin{array}{c}23.4-28.2 \\
\ldots\end{array}$ & $\begin{array}{c}23.5 \\
(23.5)\end{array}$ & $\begin{array}{c}21.3-25.1 \\
(21.3-25.1)\end{array}$ & $\begin{array}{c}24.2 \\
\ldots\end{array}$ & $\begin{array}{c}22.3-26.3 \\
\ldots\end{array}$ \\
\hline Brightest features & $\begin{array}{c}23.8 \\
(24.1)\end{array}$ & $\begin{array}{c}22.1-26.2 \\
(22.6-26.2)\end{array}$ & $\begin{array}{c}25.5 \\
\ldots\end{array}$ & $\begin{array}{c}23.4-28.0 \\
\ldots\end{array}$ & $\begin{array}{c}23.3 \\
(23.3)\end{array}$ & $\begin{array}{c}21.3-24.9 \\
(21.3-24.9)\end{array}$ & $\begin{array}{c}23.9 \\
\ldots\end{array}$ & $\begin{array}{c}22.3-26.1 \\
\ldots\end{array}$ \\
\hline
\end{tabular}
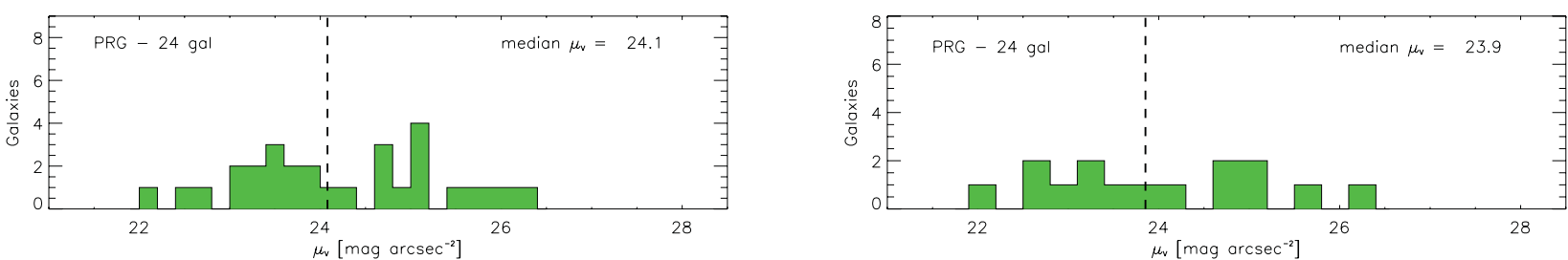

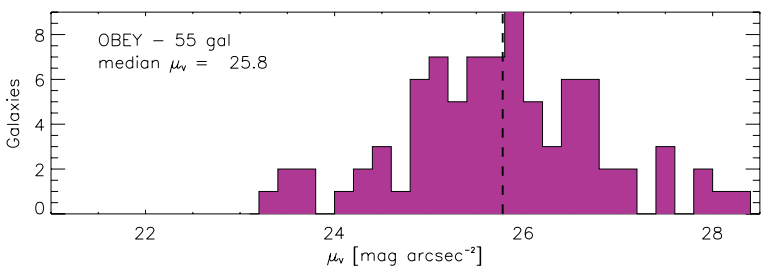

(a)

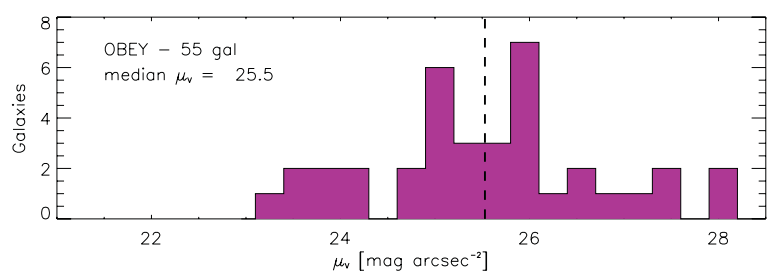

(b)

Figure 6. (a) Comparison between the $\mu_{V}^{\text {corr }}$ of the 24 PRGs in the 2 Jy sample at $z<0.2$ (top panel) and those of the elliptical galaxies in the OBEY survey (bottom panel). (b) Same as in (a) but considering the brightest feature in each source only.
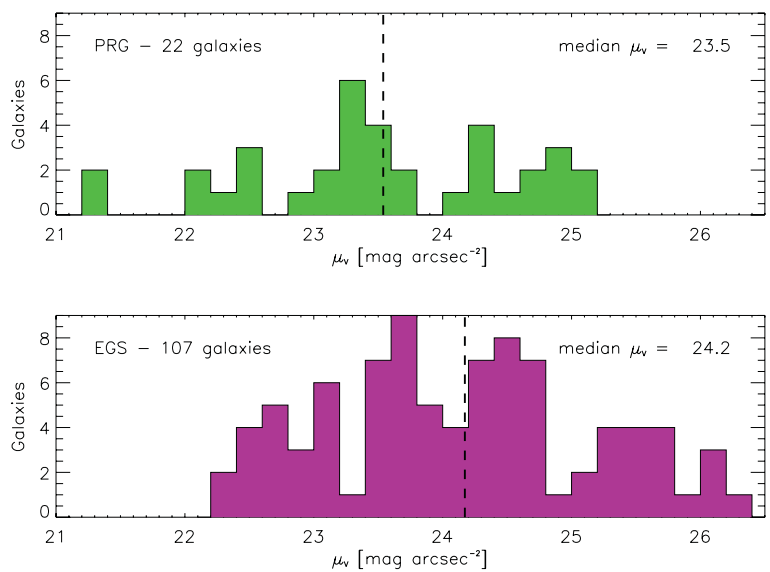

(a)
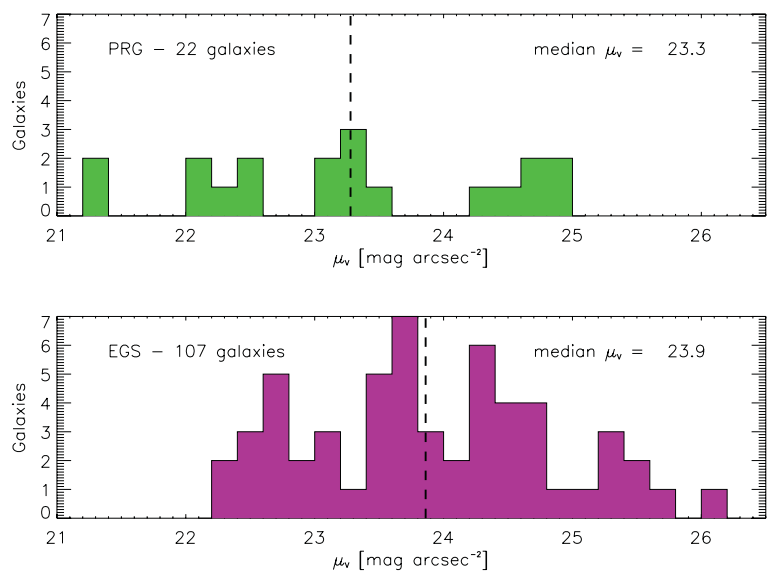

(b)

Figure 7. (a) Comparison between the $\mu_{V}^{\text {corr }}$ of the 22 PRGs in the 2Jy sample at $0.2 \leq z<0.7$ (top panel) and those of the red galaxies in the EGS sample (bottom panel). (b) Same as in (a) but considering the brightest feature in each source only.

Table 7. Percentages of disturbance found for the PRGs at $z<0.2$ and the OBEY survey at the same level surface brightness level $\left(\mu_{V}^{\text {corr }} \leq 26.2 \mathrm{mag} \mathrm{arcsec}^{-2}\right)$ and the same for the PRGs at $0.2 \leq$ $z<0.7$ and the EGS survey $\left(\mu_{V}^{\text {corr }} \leq 25.1 \mathrm{mag} \mathrm{arcsec}^{-2}\right)$. In these numbers we include galaxies classified as multiple nuclei systems (group 3). Percentages between parentheses correspond to SLRGs in the PRG sample (Columns 3 and 5) and to elliptical galaxies only in the EGS sample (column 6).

\begin{tabular}{lccccc}
\hline Morphology & Group & $\begin{array}{c}\text { PRGs } z<0.2 \\
\text { (per cent) }\end{array}$ & $\begin{array}{c}\text { OBEY } \\
\text { (per cent) }\end{array}$ & $\begin{array}{c}\text { PRGs } 0.2 \leq z<0.7 \\
\text { (per cent) }\end{array}$ & $\begin{array}{c}\text { EGS } \\
\text { (per cent) }\end{array}$ \\
\hline Signs of interaction & $1,2,3$ & $62(93)$ & 53 & $95(95)$ & $48(52)$ \\
\hline
\end{tabular}


that a significant fraction of quiescent elliptical galaxies in the two control samples show evidence for disturbed morphologies at relatively high levels of surface brightness, which are likely the result of past or on-going galaxy interactions. However, the morphological features detected in the galaxy hosts of the PRGs (e.g. tidal tails, shells, bridges, etc.) are up to 2 mag brighter than those present in their quiescent counterparts.

In fact, when we consider the same surface brightness limits for the features in the quiescent galaxies and in the PRGs (note that these limits are different in each redshift bin; see Table 7), we find that the proportion of disturbed morphologies in the quiescent population is considerably smaller ( 53 per cent at $z<0.2$ and 48 per cent at $0.2 \leq$ $z<0.7$ ) than for the PRGs (93 per cent at $z<0.2$ and 95 per cent at $0.2 \leq z<0.7$, considering SLRGs only). This indicates that galaxy interactions are likely to play a role in the triggering of the PRG activity.

However, it is important to recognize that a proportion of the quiescent elliptical galaxy population do show disturbed features at a similar level of surface brightness to the PRGs. Moreover, even if the proportion of such objects is relatively small, their volume density could be considerably larger than that of the (rare) PRGs. This raises the question of how the populations of disturbed elliptical galaxies and PRGs are related. The simplest assumption we can make is that all morphologically disturbed elliptical galaxies go through a radio-loud AGN phase at some stage in the galaxy interaction that causes the disturbed features. In this case, the total volume density of PRGs ( $\left.\rho_{\text {PRG }}\right)$ is related to the total volume density of disturbed elliptical galaxies $\left(\rho_{\mathrm{DE}}\right)$ by the following equation:

$\frac{\rho_{\mathrm{PRG}}}{\rho_{\mathrm{DE}}}=0.01\left(\frac{t_{\mathrm{PRG}}}{10 \mathrm{Myr}}\right)\left(\frac{t_{D F}}{1 \mathrm{Gyr}}\right)^{-1}$,

where $t_{\mathrm{PRG}}$ is the duty cycle of the powerful radio-loud AGN activity and $t_{\mathrm{T}}$ is the time-scale over which the tidal features associated with a particular galaxy interaction remain visible above the surface brightness limit of the observations. Typically, PRGs are expected to remain active over a period of $t_{\mathrm{PRG}} \sim 10-100 \mathrm{Myr}$ (Leahy, Muxlow \& Stephens 1989; Blundell, Rawlings \& Willott 1999; Shabala et al. 2008), while the tidal features will remain visible on a time-scale of 1 Gyr (Le Fèvre et al. 2000; Patton et al. 2002; Conselice et al. 2003; Kawata et al. 2006). Therefore we should expect the PRG to make up a fraction $\rho_{\mathrm{PRG}} / \rho_{\mathrm{DE}} \sim 0.01-0.1$ of the full population of disturbed elliptical galaxies assuming that all such galaxies go through a radio-loud phase.

A direct estimate of the volume density of PRGs can be obtained by integrating the radio luminosity function of Willott et al. (2001) above the lower radio power limit of the $0.05<z<0.72 \mathrm{Jy}$ sample $\left(P_{151 \mathrm{MHz}}^{\lim } \approx 1.3 \times 10^{25} \mathrm{~W} \mathrm{~Hz}^{-1} \mathrm{sr}^{-1}\right)$. We find $\rho_{\mathrm{PRG}}=2 \times$ $10^{-7} \mathrm{Mpc}^{-3}$ for $z=0$ and $\rho_{\mathrm{PRG}}=1 \times 10^{-6} \mathrm{Mpc}^{-3}$ for $z=0.5$ (corrected to our assumed cosmology). Similarly, an estimate of the volume density of disturbed elliptical galaxies can be made by integrating the optical luminosity function for red sequence galaxies above the lower $B$-band luminosity limit of the PRG and control samples $\left(M_{B}=-20.3 \mathrm{mag}\right)$, and then multiplying by the fraction of ellipticals with disturbed features of similar surface brightness to the $\operatorname{PRG}\left(f_{D} \sim 0.5\right)$. For low redshifts we integrate the luminosity function for red sequence galaxies from Baldry et al. (2004), obtaining $\rho_{\mathrm{DE}}=2 \times 10^{-4} \mathrm{Mpc}^{-3}$, while for higher redshifts we integrate the $z=0.5$ luminosity function of Faber et al. (2007), obtaining $\rho_{\mathrm{DE}}=$ $4 \times 10^{-4} \mathrm{Mpc}^{-3}$. By comparing the volume densities of PRGs and disturbed ellipticals estimated in this way we find: $\rho_{\mathrm{PRG}} / \rho_{\mathrm{DE}} \sim 10^{-3}$ for $z<0.2$ and $\rho_{\mathrm{PRG}} / \rho_{\mathrm{DE}} \sim 2 \times 10^{-3}$ for $z=0.5$. These proportions are considerably lower - by a factor of 5 or more - than those obtained above, based on the PRG duty cycle and the assumption that all disturbed elliptical galaxies go through a radio-loud phase ( $\left.\rho_{\mathrm{PRG}} / \rho_{\mathrm{DE}} \sim 0.01-0.1\right)$. We conclude that only a small proportion (§20 percent) of interacting giant elliptical galaxies with absolute magnitudes $M_{B}<-20.3$ mag are capable of hosting powerful radio sources with radio powers $P_{151 \mathrm{MHz}}^{\lim }>1.3 \times 10^{25} \mathrm{~W} \mathrm{~Hz}^{-1} \mathrm{sr}^{-1}$ for the requisite timescales.

Clearly, while undergoing a galaxy interaction of some type may be necessary to trigger powerful radio jets in a giant elliptical galaxy, it is not by itself sufficient. Other potentially important factors include: the degree of gas richness of the interacting galaxies, their mass ratio, the orbital parameters of the interaction, the masses of the galaxy bulges and associated supermassive $\mathrm{BHs}$, and the $\mathrm{BH}$ spin. For example, our classification of morphological disturbance in elliptical galaxies is relatively crude and does not precisely distinguish the type of galaxy interaction (e.g. whether 'wet' or 'dry', minor or major). Therefore it is possible that only a minority of the disturbed elliptical galaxies in our control samples are undergoing the precise type of interaction that leads to the triggering of the powerful radio-loud AGN activity.

Finally, we note that there is an important caveat to bear in mind when making the comparison between the PRG and control samples. We have matched the comparison samples in galaxy luminosity, redshift and depth of observations (see Section 3.1.4 on the latter), but we have not considered the environments of the galaxies. The environment may affect the comparison in two ways. First, if a specific type of galaxy interaction is required to trigger a PRG (e.g. a major, gas-rich merger), then that type of interaction may be favoured by a particular environment (e.g. group rather than cluster; see Hopkins et al. 2008b). Secondly, the tidal effects associated with high-density environments can rapidly disrupt the morphological structures (e.g. shells or ripples; Malin \& Carter 1983) that we use to classify the galaxies in our samples. Indeed, Tal et al. (2009) and Malin \& Carter (1983) explored the relationship between galaxy morphology and environment in their samples of nearby elliptical galaxies and found that the ellipticals in clusters generally appear less disturbed than those in group and field environments.

Despite the lack of quantification of the galaxy environments in this paper, previous studies of radio galaxies in the local Universe have shown that, while FRI sources (generally WLRGs) favour clusters, FRII galaxies (generally SLRGs) are found in a wide range of environments, ranging from field/group to moderately rich clusters (Prestage \& Peacock 1988; Smith \& Heckman 1990; Zirbel 1997), although there is some evidence that the environments of FRII objects become richer with redshift (e.g. Hill \& Lilly 1991). As noted in Section 2.1, the low-redshift OBEY control sample covers a mix of environments that is similar to that of the FRIIs in the local Universe, but less rich on average than that of local FRI galaxies. However, our conclusions based on the comparison with the control samples would only likely be affected by environmental issues if the control samples were more biased towards rich environments than our PRG sample. At present we have no evidence that this is the case, but the whole issue of matching control sample environments and the dependence of the degree of morphological disturbance on environment clearly warrants further investigation.

\subsection{Evolution of elliptical galaxies from $z \sim 0.7$}

By comparing the galaxies in the OBEY and EGS samples, we can study how the morphologies of elliptical galaxies evolve from redshift $z=0.7$ and compare with the predictions of galaxy evolu- 
Table 8. Results for the OBEY, EGS and PRG samples considering features with $\mu_{V} \leq 25.5 \mathrm{mag} \operatorname{arcsec}^{-2}$, to compare with those found by Malin \& Carter (1983) for a sample of 137 quiescent elliptical galaxies $(\mathrm{QE})$ at $z<0.01$. * For the PRGs at $0.2 \leq z<0.7$ the dimmest feature detected has a $\mu_{V}=$ $25.1 \mathrm{mag} \operatorname{arcsec}^{-2}$. In these percentages, we include galaxies with double nuclei as the only detected feature (group 3).

\begin{tabular}{lccccc}
\hline Work & Objects & Sample & Redshift & $\begin{array}{c}\Delta \mu_{V} \text { considered } \\
\left(\mathrm{mag}_{\operatorname{arcsec}}{ }^{-2}\right)\end{array}$ & $\begin{array}{c}\text { Signs of } \\
\text { interaction }\end{array}$ \\
\hline $\begin{array}{l}\text { Malin \& Carter (1983) } \\
\text { OBEY survey }\end{array}$ & QE & 137 & $<0.01$ & $\lesssim 25.5$ & $\sim 10$ per cent \\
2 Jy sample & QE & 55 & $<0.01$ & {$[23.3,25.5]$} & 34 per cent \\
EGS sample & 14 & $0.05-0.2$ & {$[22.1,25.5]$} & 79 per cent \\
2 Jy sample & ET & 107 & $0.2-0.7$ & {$[22.3,25.5]$} & 53 per cent (57 per cent) \\
\hline
\end{tabular}

tion models. Elliptical galaxies are the key to investigate the history of galaxy mass assembly, since they dominate the high-end of the local luminosity function. While many studies support a scenario in which old (1-4 Gyr) and massive $\left(M_{*}>10^{11} \mathrm{M}_{\odot}\right)$ ellipticals passively evolve from redshift $z \sim 1$ (e.g. Bundy et al. 2006 and references therein), others argue for a major role of dry-mergers in the build-up of the most massive ET galaxy population from $z \sim 1$ (e.g. Bell et al. 2004; Faber et al. 2007; Kaviraj et al. 2007; López-Sanjuan et al. 2011).

In Table 8, we show the percentage of disturbance found for the OBEY survey (elliptical galaxies in the local universe) and the EGS sample (ET galaxies with $0.2<z<0.7$ ) only considering features brighter than $\mu_{V}=25.5 \mathrm{mag} \operatorname{arcsec}^{-2}$. This value was chosen to match the limiting surface brightness of the features found by Malin $\&$ Carter (1983) for a sample of 137 elliptical galaxies at $z<0.01$. The latter authors used visual inspection of photographic plates to search for shells and ripples, finding that only 10 percent of the ellipticals showed these features. This percentage is considerably lower than the 34 per cent that we find for the OBEY survey when the same depth is considered, likely due to the limitations associated with the use of photographic images. In addition, in Malin \& Carter (1983) the authors were looking for sharp, shell-like features with the galaxy at the centre of curvature, rather than more asymmetric features such as fans, tails, bridges, etc. (D. Carter, private communication). At a similar surface brightness limit, and using a sample of ET galaxies selected from the Sloan Digital Sky Survey (SDSS) Stripe 82, Kaviraj (2010) reported that $\sim 34$ per cent of the galaxies show either tidal or dust features, in agreement with our findings for the OBEY survey.

The range of absolute magnitude that we are considering by putting together the galaxies in both the OBEY and EGS samples is $M_{B}=[-22.5,-20.4] \mathrm{mag}$ (see Section 2). The percentage of disturbed morphologies in the local universe is 34 percent and increases to 53 per cent at $z=[0.2,0.7]$ when the same depth is considered $\left(\mu_{V} \lesssim 25.5 \mathrm{mag} \operatorname{arcsec}{ }^{-2}\right)$. Thus, we find that a significant fraction of quiescent elliptical galaxies at low and intermediate redshifts show signatures of past interactions at relatively high levels of surface brightness, and that this fraction increases slightly with redshift. This is consistent with the idea that elliptical galaxies have undergone some evolution since $z=0.7$. However, the interactions that lead to this evolution cannot, in most cases, have noticeably modified their star formation histories and masses (Kaviraj et al. 2011; López-Sanjuan et al. 2011). This would explain why in the past the most massive elliptical galaxies were thought to passively evolve from $z=1$ to $z=0$.

\section{CONCLUSIONS}

We present the results from a comparison between the optical morphologies of a complete sample of 46 southern 2 Jy radio galaxies at intermediate redshifts $(0.05<z<0.7)$ and those of quiescent ET galaxies within the same mass and redshift ranges. Based on these results, we discuss the role of galaxy interactions in the triggering of PRGs. Our major results are as follows.

(i) We find that a significant fraction of quiescent ET galaxies across the full redshift range of our study show evidence for disturbed morphologies at relatively high levels of surface brightness, which are likely the result of past or on-going galaxy interactions.

(ii) The morphological features detected in the galaxy hosts of the 2 Jy sample of PRGs (e.g. tidal tails, shells, bridges, etc.) are up to 2 mag brighter than those present in their quiescent counterparts.

(iii) The fraction of disturbed morphologies in the quiescent population is considerably smaller ( 53 per cent at $z<0.2$ and 48 per cent at $0.2 \leq z<0.7$ ) than for PRGs (93 per cent at $z<0.2$ and 95 per cent at $0.2 \leq z<0.7$, considering SLRGs only) when the same surface brightness limits are considered.

(iv) These results support a scenario in which PRGs, which are likely triggered by interactions, represent a fleeting active phase of a subset of elliptical galaxies that have recently undergone mergers/interactions.

\section{ACKNOWLEDGMENTS}

CRA acknowledges financial support from STFC PDRA (ST/G001758/1). CRA, PGPG and GB acknowledge the Spanish Ministry of Science and Innovation (MICINN) through project Consolider-Ingenio 2010 Program grant CSD2006-00070: First Science with the GTC (http://www.iac.es/consolider-ingenio-gtc/). PGPG and GB acknowledge support from the Spanish Programa Nacional de Astronomía y Astrofísica under grants AYA200910368 and AYA2009-07723-E. KJI is supported through the Emmy Noether programme of the German Science Foundation (DFG). This work has made use of the Rainbow Cosmological Surveys Data base, which is operated by the Universidad Complutense de Madrid (UCM). This work is based in part on data collected at Subaru Telescope, which is operated by the National Astronomical Observatory of Japan. This research has made use of the NED which is operated by the Jet Propulsion Laboratory, California Institute of Technology, under contract with the National Aeronautics and Space Administration. The authors specially acknowledge Tomer Tal for providing access to the OBEY images, as well as David 
Carter, Richard Pogge, Philip Massey, Giovanni Carraro and Carlos López-SanJuan for their valuable help. We finally acknowledge useful comments from the anonymous referee.

\section{REFERENCES}

Allen S. W., Dunn R. J. H., Fabian A. C., Taylor G. B., Reynolds C. S., 2006, MNRAS, 372, 21

Athey A., Bregman J. N., Bergman J. D., Temi P., Sauvage M., 2002, ApJ, 571,272

Baldry I. K., Glazebrook K., Brinkmann J., Ivezić Z̆., Lupton R. H., Nichol R. C., Szalay A. S., 2004, ApJ, 600, 681

Balmaverde B., Baldi R. D., Capetti A., 2008, A\&A, 486, 119

Barro G. et al., 2009, A\&A, 494, 63

Barro G. et al., 2011, ApJS, 193, 13

Bell E. F. et al., 2004, ApJ, 608, 752

Bell E. F. et al., 2006, ApJ, 640, 241

Bennert N., Canalizo G., Jungwiert B., Stockton A., Schweizer F., Peng C. Y., Lacy M., 2008, ApJ, 677, 846

Best P. N., Kaiser C. R., Heckman T. M., Kauffmann G., 2006, MNRAS, 368, L67

Blanton M. R., 2006, ApJ, 648, 268

Blundell K. M., Rawlings S., Willott C. J., 1999, AJ, 117, 677

Borne K. D., 1984, ApJ, 287, 503

Bundy K. et al., 2006, ApJ, 651, 120

Bundy K. et al., 2010, ApJ, 719, 1969

Buttiglione S., Capetti A., Celotti A., Axon D. J., Chiaberge M., Macchetto F. D., Sparks W. B., 2010, A\&A, 509, 6

Canalizo G., Stockton A., 2000, ApJ, 528, 201

Canalizo G., Stockton A., 2001, ApJ, 555, 719

Canalizo G., Bennert N., Jungwiert B., Stockton A., Schweizer F., Lacy M., Peng C., 2007, ApJ, 669, 801

Cardelli J. A., Clayton G. C., Mathis J., 1989, ApJ, 345, 245

Cattaneo A., Combes F., Colombi S., Bertin E., Melchior A.-L., 2005, MNRAS, 359, 1237

Cimatti A., Daddi E., Renzini A., 2006, A\&A, 453, L29

Cisternas M. et al., 2011, ApJ, 726, 57 (C11)

Conselice C. J., Bershady M. A., Dickinson M., Papovich C., 2003, AJ, 126, 1183

Cox T. J., Jonsson P., Primack J. R., Somerville R. S., 2006, MNRAS, 373 , 1013

Cox T. J., Jonsson P., Somerville R. S., Primack J. R., Dekel A., 2008, MNRAS, 384, 386

Croton D. J. et al., 2006, MNRAS, 365, 11

Davis M. et al., 2007, ApJ, 660, 1

de Koff S., Baum S. A., Sparks W. B., Biretta J., Golombek D., Macchetto F., McCarthy P., Miley G. K,. 1996, ApJS, 107, 621

de Koff S. et al., 2000, ApJS, 129, 33

de Vaucouleurs G., de Vaucouleurs A., Corwin H. G., Jr, Buta R. J., Paturel G., Fouque P., 1991, Third Reference Catalogue of Bright Galaxies. Springer, Berlin

Di Matteo T., Springel V., Hernquist L., 2005, Nat, 433, 604

di Matteo P., Combes F., Melchior A.-L., Semelin B., 2007, A\&A, 468, 61

di Serego Alighieri S., Danziger I. J., Morganti R., Todhunter C. N., 1994, MNRAS, 269, 998

Dicken D., Tadhunter C., Axon D., Morganti R., Inskip K. J., Holt J., González Delgado R., Groves B., 2009, ApJ, 694, 268

Dunlop J. S., McLure R. J., Kukula M. J., Baum S. A., O’Dea C. P., Hughes D. H., 2003, MNRAS, 340, 1095

Edge A. C., Ivison R. J., Smail, Ian, Blain A. W., Kneib J.-P., 1999, MNRAS, 306, 599

Edge A. C. et al., 2010, A\&A, 518, L47

Ellison S. L., Patton D. R., Trevor Mendel J., Scudder J. M., 2011, MNRAS, in press (doi:10.1111/j.1365-2966.2011.19624.x)

Faber S. M. et al., 2007, ApJ, 665, 265

Fabian A. C., Johnstone R. M., Daines S. J., 1994, MNRAS, 271, 737

Feldmann R., Mayer L., Carollo C. M., 2008, ApJ, 684, 1062

Ferrarese L., Merritt D., 2000, ApJ, 539, L9
Frei Z., Gunn J. E., 1994, AJ, 108, 1476

Fukugita M., Shimasaku K., Ichikawa T., 1995, PASP, 107, 945

Gabor J. M. et al., 2009, ApJ, 691, 705

Gebhardt K. et al., 2000, ApJ, 539, L13

Georgakakis A. et al., 2009, MNRAS, 397, 623

Goudfrooij P., Hansen L., Jørgensen H. E., Nørgaard-Nielsen H. U., 1994, A\&AS, 105, 341

Graham A. W., 2008, ApJ, 680, 143

Graham A. W., Onken C. A., Athanassouia E., Combes F., 2011, MNRAS, 412,2211

Greene J. E., Ho L. C., 2006, ApJ, 641, L21

Grogin N. A. et al., 2005, ApJ, 627, L97

Groth E. J., Kristian J. A., Lynds R., O’Neil E. J., Jr, Balsano R., Rhodes J., 1994, BAAS, 26, 1403

Hansen L., Jorgensen H. E., Norgaard-Nielsen H. U., 1995, A\&AS, 297, 13

Hardcastle M. J., Evans D. A., Croston J. H., 2007, MNRAS, 376, 1849

Heckman T. M., Smith E. P., Baum S. A., van Breugel W. J. M., Miley G. K., Illingworth G. D., Bothun G. D., Balick B., 1986, ApJ, 311, 526

Heckman T. M., Kauffmann G., Brinchmann J., Charlot S., Tremonti C., White S. D. M., 2004, ApJ, 613, 109

Hernquist L., Spergel D. L., 1992, ApJ, 399, L117

Hill G. J., Lilly S. J., 1991, ApJ, 367, 1

Hoessel J. G., 1980, ApJ, 241, 493

Hook I., Jørgensen I., Allington-Smith J. R., Davies R. L., Metcalfe N., Murowinski R. G., Crampton D., 2004, PASP, 116, 425

Hopkins P. F., Cox T. J., Kereš D., Hernquist L., 2008a, ApJS, 175, 390

Hopkins P. F., Hernquist L., Cox T. J., Kereš D., 2008b, ApJS, 175, 356

Hu J., 2008, MNRAS, 386, 2242

Hutchings J. B., 1987, ApJ, 320, 122

Kauffmann G., Haehnelt M., 2000, MNRAS, 311, 576

Kaviraj S., 2010, MNRAS, 406, 382

Kaviraj S. et al., 2007, ApJS, 173, 619

Kaviraj S., Tan K.-M., Ellis R. S., Silk J., 2011, MNRAS, 411, 2148

Kaviraj S. et al., 2011, MNRAS, submitted (arXiv:1107.5306)

Kawata D., Mulchaey J. S., Gibson B. K., Sánchez-Blázquez P., 2006, ApJ, 648, 969

Keel W. C., 1996, AJ, 111, 696

Knaap G. R., Guhathakurta P., Kim D.-W., Jura M., 1989, ApJS, 70, 329

Kormendy J., Richstone D., 1995, ARA\&A, 33, 581

Kormendy J., Bender R., Cornell M. E., 2011, Nat, 469, 374

Koss M., Mushotzky R., Veilleux S., Winter L., 2010, ApJ, 716, L125

Kuo C.-Y., Lim J., Tang Y.-W., Ho P. T. P., 2008, ApJ, 679, 1047

Leahy J. P., Muxlow T. W. B., Stephens P. W., 1989, MNRAS, 239, 401

Le Fèvre O. et al., 2000, MNRAS, 311, 565

López-Sanjuan C. et al., 2011, A\&A, 530, 20

Lotz J. M., Jonsson P., Cox T. J., Primack J. R., 2008, MNRAS, 391, 1137

McIntosh D. H., Guo Y., Hertzberg J., Katz N., Mo H. J., van den Bosch F. C., Yang X., 2008, MNRAS, 388, 1537

Magorrian J. et al., 1998, AJ, 115, 2285

Malin D. F., Carter D., 1983, ApJ, 274, 534

Malkan M. A., Gorjian V., Tam R., 1998, ApJ, 117, 25

Michard R., 2005, A\&A, 441, 451

Miyazaki S. et al., 2002, PASJ, 54, 833

Naab T., Khochfar S., Burkert A., 2006, ApJ, 636, L81

Park S. Q. et al., 2008, ApJ, 678, 744

Patton D. R. et al., 2002, ApJ, 565, 208

Pérez-González P. G., Trujillo I., Barro G., Gallego J., Zamorano J., Conselice C. J., 2008, ApJ, 687, 50

Prestage R. M., Peacock J. A., 1988, MNRAS, 230, 131

Prugniel P., Heraudeau P., 1998, VizieR Online Data Catalog, 7206, 0

Quinn P. J., 1984, ApJ, 279, 596

Ramos Almeida C., Tadhunter C. N., Inskip K. J., Morganti R., Holt J., Dicken D., 2011, MNRAS, 410, 1550 (RA11) 
Schlegel D. J., Finkbeiner D. P., Davis M., 1998, ApJ, 500, 525

Shabala S. S., Ash S., Alexander P., Riley J. M., 2008, MNRAS, 388, 625

Smith E. P., Heckman T. M., 1989, ApJ, 341, 658

Smith E. P., Heckman T. M., 1990, ApJ, 348, 38

Somerville R. S., Hopkins P. F., Cox T. J., Robertson B. E., Hernquist L., 2008, MNRAS, 391, 481

Springel V., Di Matteo T., Hernquist L., 2005, MNRAS, 361, 776

Tadhunter C. N., Morganti R., di Serego-Alighieri S., Fosbury R. A. E., Danziger I. J., 1993, MNRAS, 263, 999

Tadhunter C. N., Morganti R., Robinson A., Dickson R., Villar-Martín M., Fosbury R. A. E., 1998, MNRAS, 298, 1035

Tadhunter C. et al., 2011, MNRAS, 412, 960

Tal T., van Dokkum P. G., Nelan J., Bezanson R., 2009, AJ, 138, 1417
Tully R. B., 1988, Nearby Galaxies Catalog. Cambridge Univ. Press, Cambridge, p. 221

van Dokkum P. G., 2005, ApJ, 130, 2647

van Dokkum P. G., Franx M., 1995, AJ, 110, 2027

Wall J. V., Peacock J. A., 1985, MNRAS, 216, 179

Wild V., Heckman T., Charlot S., 2010, MNRAS, 405, 933

Willott C. J., Rawlings S., Blundell K. M., Lacy M., Eales S. A., 2001, MNRAS, 322, 536

Zhao Y.-H., Huang J.-S., Ashby M., Fazio G., Miyazaki S., 2009, Res. Astron. Astrophys., 9, 1061

Zirbel E. L., 1997, ApJ, 476, 489

This paper has been typeset from a $\mathrm{T}_{\mathrm{E}} \mathrm{X} / \mathrm{LT} \mathrm{E} \mathrm{X}$ file prepared by the author. 